\title{
FREE STATES OF THE GAUGE INVARIANT CANONICAL ANTICOMMUTATION RELATIONS
}

\author{
BY
}

B. M. BAKER

\begin{abstract}
ABSTRACr. The gauge invariant subalgebra of the canonical anticommutation relations (henceforth GICAR) is viewed as an inductive limit of finitedimensional $C^{*}$-algebras, and a study is made of a simple class of its representations. In particular, representations induced by restricting the wellknown gauge invariant generalized free states from the entire canonical anticommutation relations (henceforth CAR) are considered. Denoting (a) a state of the CAR by $\omega$ and its restriction to the GICAR by $\omega^{\circ}$, (b) the unique gauge invariant generalized free state of the CAR such that $\omega\left(a(f)^{*} a(g)\right)$ $=(f, A g)$ by $\omega_{A}$, it is shown that (1) $\omega_{A}^{\circ}$ induces (an impure) factor representation of the GICAR if and only if $\operatorname{Tr} A(I-A)=\infty$, (2) two (impure) GICAR factor representations $\omega_{A}^{\circ}$ and $\omega_{B}^{\circ}$ are quasi-equivalent if and only if $A^{1 / 2}-B^{1 / 2}$ and $(I-A)^{1 / 2}-(I-B)^{1 / 2}$ are Hilbert-Schmidt class operators.
\end{abstract}

1. Introduction. In this work we study the gauge invariant ${ }^{1}$ subalgebra of the canonical anticommutation relations via a simple class of its representations. Following [13] and [4] we view the canonical anticommutation relations (henceforth CAR) and its $U(1)$ or gauge invariant subalgebra (henceforth GICAR) as $C^{*}$-algebras which are in fact limits of ascending sequences of finite-dimensional matrix algebras; in the terminology of [6] and [4] they are examples of uniformly hyperfinite (UHF) and approximately finite (AF) algebras respectively. We then proceed to examine the representations induced by restricting the gauge invariant generalized free states of the CAR (see \$2) to the GICAR. Previously, many authors have studied UHF algebras in general (e.g. [6], [11]) and the generalized free states of the CAR in particular, as in [1], [2], [5], [12], and [13]. AF algebras (including the GICAR) are introduced and studied in [4].2

Received by the editors April 20, 1976.

AMS (MOS) subject classifications (1970). Primary 46L05, 81A17; Secondary 81A81.

Key words and phrases. Anticommutation relations, gauge invariance, approximately finite $C^{*}$. algebra, generalized free states, factor representations, quasi-equivalent representations.

1 In field theory, automorphisms induced by the action of $U(1)$ on the field algebra are called gauge transformations of the first kind.

2 In [4] the GICAR is referred to as the fermion current algebra, following [9]. 
The present work is motivated by [7]-[9] where $C^{*}$-algebras of observables are studied in general, and that of [1], [2], [5], and [12] in which techniques of field theory are used to analyze the generalized free states of the CAR. Throughout, the generalizations of the work of [6] and [11] on UHF algebras to the AF case accomplished in [4] are taken as the starting point. The main result of \$3, a necessary and sufficient condition for a gauge invariant generalized free state of the CAR to induce a factor representation of the GICAR by restriction, naturally extends the results of [14]. The main result of $\$ 4$, a necessary and sufficient condition for two factor states of the GICAR to induce quasi-equivalent representations, is identical in conclusion to Theorem 5.1 of [12], whose techniques and results are relied upon heavily.

I would like to thank my thesis advisor, Robert T. Powers. Without his many helpful suggestions this work could not have been done. I also thank Herbert Wilf for his statement and proof of Proposition 3.19.

2. Definitions, notation. Let $\Re$ be a separable Hilbert space with orthonormal basis $\left\{f_{n}\right\}, n=1,2, \ldots$, and let $\Re_{n}=\operatorname{span}\left\{f_{1}, \ldots, f_{n}\right\}$. We denote by $\mathfrak{Q}\left(\pi_{n}\right)$ the CAR algebra over $\Re_{n}$. As sketched below, this is constructed via a linear mapping $f \rightarrow a(f)$ satisfying the relations

$$
\begin{gathered}
a(f) a(g)+a(g) a(f)=0, \\
a(f) a(g)^{*}+a(g)^{*} a(f)=(g, f) I,
\end{gathered}
$$

for all $f, g$ in $\Re_{n}$. We denote by $\mathbb{Q}(\mathcal{K})$ the CAR algebra over $\mathscr{K}$, the completion of $\cup_{n} \mathfrak{Q}\left(\Re_{n}\right)$, written $\mathscr{Q}(\mathscr{K})=\overline{\cup_{n} \mathscr{Q}\left(\Re_{n}\right)}$. This is a UHF algebra in the sense of [6].

Setting

$$
\mathfrak{B}_{1}=\mathfrak{Q}\left(\Re_{1}\right), \quad \mathscr{B}_{k}=\mathfrak{Q}\left(\Re_{k}\right) \cap \mathbb{Q}\left(\Re_{k-1}\right)^{c}, \quad k=2,3, \ldots,
$$

we may choose matrix units for these algebras as follows:

$$
\begin{array}{ll}
e_{11}^{(k)}=a\left(f_{k}\right) a\left(f_{k}\right)^{*}, & e_{12}^{(k)}=a\left(f_{k}\right) V_{k}, \\
e_{21}^{(k)}=a\left(f_{k}\right)^{*} V_{k}, & e_{22}^{(k)}=a\left(f_{k}\right)^{*} a\left(f_{k}\right),
\end{array}
$$

where $V_{1}=I, \quad V_{k}=\Pi_{j=1}^{k-1}\left(I-2 a\left(f_{j}\right)^{*} a\left(f_{j}\right)\right), \quad k=2,3, \ldots$ It follows straightforwardly that (1) each $\mathscr{B}_{k}$ is isomorphic to a $2 \times 2$ matrix algebra, (2) the $\left\{\mathscr{B}_{k}\right\}, k=1,2, \ldots$, commute pairwise for differing $k$ and $(3) \mathbb{Q}\left(\Re_{n}\right)$ is isomorphic to $\otimes_{k=1}^{n} \mathfrak{B}_{k}$, written $\mathbb{Q}\left(\Re_{n}\right) \approx \otimes_{k=1}^{n} \mathfrak{B}_{k}$. From (3) we conclude $\mathbb{Q}\left(\pi_{n}\right)$ isomorphic to a $2^{n} \times 2^{n}$ matrix algebra with matrix units 


$$
e_{i_{1} j_{1}}^{(1)} \cdot e_{i_{2} j_{2}}^{(2)} \cdots \cdots e_{i_{n} j_{n}}^{(n)}, \quad 1 \leqslant i_{k}, j_{k} \leqslant n, k=1, \ldots, n .
$$

We denote by $\mathscr{Q}^{\circ}(\mathcal{K})$ the $U(1)$ or gauge invariant subalgebra of $\mathscr{Q}(\mathcal{K})$. More explicitly, the mapping $a(f) \rightarrow e^{-i t} a(f)$ is extended to an automorphism of $\mathbb{Q}(\mathscr{K})$, which we denote $\chi_{t}$, and we define $\mathbb{Q}^{\circ}\left(\Re_{n}\right)=\left\{x\right.$ in $\mathbb{Q}\left(\Re_{n}\right): \chi_{t}(x)=x$, $0 \leqslant t<2 \pi\}$. It follows that $\mathbb{Q}^{\circ}\left(\Re_{n}\right) \approx \oplus_{k=0}^{n} M_{(n k)}$ with $M_{(n k)}$ an $\left(\begin{array}{l}n \\ k\end{array}\right) \times\left(\begin{array}{l}n \\ k\end{array}\right)$ matrix algebra and

$$
\left(\begin{array}{l}
n \\
k
\end{array}\right)=\frac{n !}{k !(n-k) !}
$$

(see [4]). Finally, we define $\mathscr{Q}^{\circ}(\Re)=\overline{U_{n} \mathbb{Q}^{\circ}\left(\Re_{n}\right)}$, an AF algebra in the sense of [4].

In the sequel we will investigate a simple class of representations of $\mathscr{Q}^{\circ}(\mathcal{K})$ obtained by restricting the gauge invariant generalized free states of $\mathscr{Q}(\mathcal{C})$ to $\mathfrak{Q}^{\circ}(\mathcal{K}) \subset \mathbb{Q}(\mathscr{K})$. In general, a state of $\mathbb{Q}(\mathscr{K})$ is determined by its values on monomials of the form $a\left(f_{n}\right)^{*} \cdots a\left(f_{1}\right)^{*} a\left(g_{1}\right) \cdots a\left(g_{m}\right)$ since (1) a state is linear and continuous, (2) polynomials in the $a(f)^{*}, a(g)$ with $f, g$ in $\mathcal{K}$ are norm dense in $\mathscr{Q}(\mathscr{K})$, (3) polynomials may be written as a combination of monomials in the indicated form via the anticommutation relations. In particular, a state $\omega$ of $\mathscr{Q}(\mathcal{K})$ is called a generalized free state if it vanishes on monomials odd in the $a(f), a(g)^{*}$ and its truncated $N$-point functions (see, e.g., [10]) vanish for $N>2$; it follows from the latter property that $\omega$ is completely determined by its 2-point functions $\omega\left(a(f)^{*} a(g)\right)$ and $\omega(a(f) a(g))$. Here we consider gauge invariant generalized free states; these are determined by the single 2-point function $\omega\left(a(f)^{*} a(g)\right)$ which by linearity and boundedness properties can be written as $(f, A g)$ for some linear operator $A, 0 \leqslant A$ $\leqslant I$. More precisely we define the gauge invariant generalized free state $\omega_{A}$ of $Q(\mathcal{K})$ by the formula

$$
\omega_{A}\left(a\left(f_{n}\right)^{*} \cdots a\left(f_{1}\right)^{*} a\left(g_{1}\right) \cdots a\left(g_{m}\right)\right)=\delta_{n m} \operatorname{det}\left(\left(f_{i}, A g_{j}\right)\right) .
$$

We denote by $\omega_{A}^{\circ}$ the restriction of $\omega_{A}$ to $\mathcal{Q}^{\circ}(\mathcal{K})$.

A state $\omega$ of $\mathscr{Q}(\mathcal{K})$ is said to factorize with respect to the $\left\{\mathscr{B}_{k}\right\}, k=1,2, \ldots$, or $\left\{\Re_{k}\right\}, k=1,2, \ldots$, defined above, if $\omega(x y)=\omega(x) \omega(y)$ whenever $x$ is in $\mathscr{B}_{l}$ and $y$ is in $\mathscr{B}_{m}, m \neq l$. Two states $\omega_{1}$ and $\omega_{2}$ are said to be unitarily equivalent, denoted $\omega_{1} \sim_{u} \omega_{2}$, if they induce unitarily equivalent representations. Two states are said to be quasi-equivalent, denoted $\omega_{1} \widetilde{q}_{q} \omega_{2}$, if they induce quasi-equivalent representations; letting $\Pi_{1}\left(\Pi_{2}\right)$ be the representation induced by $\omega_{1}\left(\omega_{2}\right)$ this means there is an isomorphism $h$ between the von Neumann algebras $\Pi_{1}^{\prime \prime}$ and $\Pi_{2}^{\prime \prime}$ such that $h\left(\Pi_{1}(x)\right)=\Pi_{2}(x)$ for all $x$ in $\mathcal{Q}(\mathcal{K})$.

For convenience, we summarize some results on gauge invariant generalized free states, giving an appropriate reference for each. 
STATEMENT

Equivalent Condition

REFERENCE

CAR

$\omega_{A}$ is a pure state

$A$ is a projection

$\omega_{E} \sim \omega_{F}(E, F$ are projections) $E-F$ is Hilbert-Schmidt class

$\omega_{A}$ is a factor state

automatic

$\omega_{A} \sim_{q} \omega_{B}$

$A^{1 / 2}-B^{1 / 2}$ and $(I-A)^{1 / 2}-(I-B)^{1 / 2}$

are Hilbert-Schmidt class

\section{GICAR}

$\omega_{A}^{\circ}$ is a pure state

$A$ is a projection

$\omega_{E}^{\circ} \sim_{u} \omega_{F}^{\circ}$

$\operatorname{Tr}((I-E) F(I-E))$

( $E, F$ are projections)

$$
=\operatorname{Tr}((I-F) E(I-F))<\infty
$$

$\omega_{A}^{\circ}$ is a factor state

$\operatorname{Tr} A(I-A)=\infty$

$\omega_{A}^{\circ} \sim_{q} \omega_{B}^{\circ}$

$A^{1 / 2}-B^{1 / 2}$ and $(I-A)^{1 / 2}-(I-B)^{1 / 2} \S 4$

are Hilbert-Schmidt class

We note that nongauge invariant generalized free states are studied in [1], [3], and [5]. For further elaboration on notation, definitions, or results we ask the reader to consult the references, especially [10].

3. Factor condition. In this section, we wish to prove

THEOREM. Let $A$ be an operator on a Hilbert space $\mathscr{H}$, with $0<A<I$. Suppose $A$ is not a projection. Let $\omega_{A}^{\circ}$ be the associated generalized free state of $\mathbb{Q}^{\circ}(\mathcal{F})$. Then $\omega_{A}^{\circ}$ is a factor state if and only if $\operatorname{Tr} A(I-A)=\infty$.

We note that when $A$ is a projection, it can be shown that $\omega_{A}^{\circ}$ is a pure state, as in [14, III, 1, Proposition]. Our method of attack will be to eventually apply the following theorem.

THEOREM 3.1 [4, THEOREM 4.4]. Let $\mathfrak{Q}=\overline{U_{n} \mathbb{Q}_{n}}$ and suppose that $\omega$ is a state of $Q$ and $\Pi_{\omega}$ the representation associated to $\omega$ by the Gel'fand-Segal construction. Then the following conditions are equivalent:

(i) $\omega$ is a factor state.

(ii) For all $x$ in $\mathbb{Q}$ there exists an integer $r>0$ such that $|\omega(x y)-\omega(x) \omega(y)|$ $\leqslant\left\|\Pi_{\omega}(y)\right\|$ for all $y$ in $\mathbb{Q}_{r}^{c}$.

(iii) For all $x$ in $\mathbb{Q}$ there exists a finite-dimensional *-algebra $\mathfrak{B} \subseteq \mathbb{Q}$ containing e such that $|\omega(x y)-\omega(x) \omega(y)| \leqslant\left\|\Pi_{\omega}(y)\right\|$ for all $y$ in $\mathfrak{B}^{c}$.

In order to use condition (ii) above, for the case $\mathscr{Q}=\mathscr{Q}^{\circ}(\mathcal{K})=\overline{U_{r} \mathbb{Q}^{\circ}\left(\mathscr{T}_{r}\right)}$, we will examine the relative commutant of $\mathscr{Q}^{\circ}\left(\mathscr{R}_{r}\right)$ in $\mathscr{Q}^{\circ}(\mathscr{T})$. (Here it will be necessary to distinguish between the relative commutant in $\mathbb{Q}^{\circ}(\mathcal{F})$ and the relative commutant in $\mathscr{Q}(\mathcal{K})$. Thus we will use the superscripts $c_{0}$ for the 
former and $c$ for the latter.) The Lemmas 3.2-3.10 below, together with Proposition 3.11, provide us with a useful form for $\mathbb{Q}^{\circ}\left(\Re_{r}\right)^{c_{0}}$.

LEMMA 3.2. Let Th be a finite-dimensional Hilbert space with orthonormal basis $\left\{f_{1}, \ldots, f_{n}\right\}$, and

$$
N(\Re)=\sum_{i=1}^{n} a\left(f_{i}\right)^{*} a\left(f_{i}\right)=\sum_{i=1}^{n} e_{22}^{(i)}
$$

Then $x$ in $\mathbb{Q}(\Re)$ commutes with $N(\Re)$ if and only if $x$ is in $\mathbb{Q}^{\circ}(\Re)$.

Proof. First recall that $\mathcal{Q}(\Re)$ is a $p \times p$ matrix algebra with $p=2^{n}$. Letting $\Phi, \psi$ be index functions such that $\Phi, \psi:\{1, \ldots, n\} \rightarrow\{1,2\}$ we define

$$
f_{\Phi \psi}=e_{\Phi(1) \psi(1)}^{(1)} e_{\Phi(2) \psi(2)}^{(2)} \cdots \cdots e_{\Phi(n) \psi(n)}^{(n)}
$$

which is a matrix unit for $\mathcal{Q}^{\circ}(\Re)$ if and only if $\Phi$ and $\psi$ take on the value 2 (and hence 1) the same number of times, i.e., $\sum_{j=1}^{n} \psi(i)=\sum_{i=1}^{n} \Phi(i)$. Now note

$$
\begin{aligned}
& {\left[\sum_{\Phi, \psi} c_{\Phi, \psi} f_{\Phi, \psi}, N(\Re)\right]_{-}=\sum_{\Phi \psi} c_{\Phi \psi} \sum_{i}\left[f_{\Phi \psi}, e_{22}^{(i)}\right]} \\
& =\sum_{\Phi \psi} c_{\Phi \psi}\left(\sum_{i}(\psi(i)-\Phi(i))\right) f_{\Phi \psi}=0 \Leftrightarrow c_{\Phi \psi}=0
\end{aligned}
$$

for all $\Phi, \psi$ such that $\Sigma_{i} \Phi(i) \neq \Sigma_{i} \psi(i)$. Since any $x$ in $\mathbb{Q}^{\circ}(\Re)$ may be written as such a sum, we have $x$ in $\mathbb{Q}^{\circ}(\mathscr{N}) \Leftrightarrow[x, N(\mathscr{N})]_{-}=0$. Done.

LEMMA 3.3. Let $\Re$ be a separable Hilbert space and $\Re \subset \Re$ a finitedimensional subspace. Let $N(\Re)$ be as above, and c denote the commutant relative to $\mathbb{Q}(\mathscr{K})$. Then

$$
\mathbb{Q}^{\circ}(\Re)=\mathbb{Q}(\Re) \wedge N(\Re)^{c} .
$$

Proof. Immediate, from Lemma 3.2.

LEMMA 3.4. Let $z\left(\mathbb{Q}^{\circ}(\Re)\right)$ denote the center of $\mathbb{Q}^{\circ}(\Re)$ and $\mathbb{Q}(N(\Re))$ the $C^{*}$ algebra generated by the single element $N(\mathscr{T})$. Then $z\left(\mathbb{Q}^{\circ}(\mathscr{T})\right)=\mathbb{Q}(N(\mathscr{T}))$.

Proof. From Lemma 3.3 we have $\mathbb{Q}^{\circ}(\mathscr{T})=\mathscr{Q}(\mathscr{T}) \wedge N(\mathscr{T})$. Setting $\mathcal{K}=\mathscr{N}$, we have $c$ denoting the commutant relative to $\mathcal{Q}(\mathscr{N})$. Taking the commutant we obtain $\mathbb{Q}^{\circ}(\mathscr{N})^{c}=\mathscr{Q}(\mathfrak{N})^{c} \vee N(\mathscr{N})^{c c}$ recalling that for von Neumann algebras $R_{1}$ and $R_{2},\left(R_{1} \wedge R_{2}\right)^{\prime}=R_{1}^{\prime} \vee R_{2}^{\prime}$. By the double commutant theorem we have $N(\mathscr{N})^{c c}=\mathscr{Q}(N(\mathscr{N}))$, and since $\mathscr{Q}(\mathscr{N})$ is a factor we conclude

$$
\mathbb{Q}^{\circ}(\mathscr{T})^{c}=\{\lambda I\} \vee \mathbb{Q}(N(\mathscr{T}))=\mathbb{Q}(N(\mathscr{T})) .
$$


Now $N(\mathscr{T})$ is in $\mathbb{Q}^{\circ}(\Re)$ so $\mathscr{Q}(N(\Re)) \subset \mathbb{Q}^{\circ}(\Re)$; hence $z\left(\mathscr{Q}^{\circ}(\Re)\right)=\mathscr{Q}^{\circ}(\Re)^{c}$ $\wedge \mathbb{Q}^{\circ}(\Re)=\mathbb{Q}(N(\Re)) \wedge \mathbb{Q}^{\circ}(\Re)=\mathbb{Q}(N(\Re))$. Done.

LEMma 3.5 [12, LemMa 2.2]. Let $\Re$ be a Hilbert space, and $\Re \subset \nVdash$ a finitedimensional subspace with orthonormal basis $\left\{f_{1}, \ldots, f_{n}\right\}$. Let

$$
V=\prod_{i=1}^{n}\left(I-2 a\left(f_{i}\right)^{*} a\left(f_{i}\right)\right)
$$

and let $c$ denote the commutant relative to $\mathbb{Q}(\mathscr{K})$. Then $\mathbb{Q}(\mathfrak{T})^{c}$ is generated by the elements $a(f) V$ for all $f$ in $\Re^{\perp}$.

LEMMA 3.6. Let $\Re$ and $\Re$ be finite-dimensional Hilbert spaces with $\Re \subset \Re$. Let $c$ denote the commutant relative to $\mathbb{Q}(\Re)$. Then $\mathbb{Q}^{\circ}(\Re)^{c}=\mathbb{Q}\left(\pi^{\perp}\right)$ $\vee N(\mathscr{R})$.

Proof. From Lemma 3.3 we have $\mathscr{Q}^{\circ}(\Re)=\mathscr{Q}(\Re) \wedge N(\Re)^{c}$, and taking the commutant we get $\mathbb{Q}^{\circ}(\pi)^{c}=\mathscr{Q}(\pi)^{c} \vee N(\Re)^{c c}=\mathscr{Q}(\Re)^{c} \vee N(\Re)$. By the above lemma, we have

$$
\mathbb{Q}^{\circ}(\Re)^{c}=\mathscr{Q}\left(\left\{a(f) V, f \text { in } \Re^{\perp}\right\}\right) \vee N(\Re)=\mathscr{Q}\left(\Re^{\perp}\right) \vee N(\Re)
$$

since $V=\exp (i \pi N(\mathscr{R}))$ is in $\mathscr{Q}(N(\mathscr{N}))$. Done.

LEMma 3.7. Let $\Re$ be a Hilbert space and $\Re \subset \Re$ a finite-dimensional subspace. Let $c$ denote the commutant relative to $\mathbb{Q}(\mathscr{K})$. Then $\mathbb{Q}^{\circ}(\Re)^{c}=\mathbb{Q}\left(\Re^{\perp}\right)$ $\vee N(\Re)$.

Proof. (つ) As in Lemma 3.5, for $f$ in $\Re^{\perp}$ we have $a(f) V$ an element of $\mathbb{Q}(\pi)^{c} \subset \mathbb{Q}^{\circ}(\Re)^{c}$. Further $N(\Re)$ and $V=\exp (i \pi N(\Re))$ are in $\mathbb{Q}^{\circ}(\Re)^{c}$ by Lemma 3.2. Since $V^{2}=I$ we conclude such $a(f)$ to be elements of $Q^{\circ}(\Re)^{c}$. Hence $\mathscr{Q}\left(\Re^{\perp}\right) \vee N(\Re) \subset \mathbb{Q}^{\circ}(\Re)^{c}$. Done.

(C) Let $x$ be in $\mathbb{Q}^{\circ}(\Re)^{c}$, and let $\left\{\Re_{k}\right\}, k=1,2, \ldots$, be an increasing sequence of subspaces of $\Re$ such that $\Re=\Re_{1}$ and $\bar{U}_{n} \Re_{n}=\Re$. Certainly, $x$ is in $\mathfrak{Q}(\mathscr{K})=\overline{\cup_{n} \mathscr{Q}\left(\Re_{n}\right)}$; hence there exists a sequence $\left\{x_{n}\right\}, n=1,2, \ldots$, such that $x_{n}$ is in $\mathbb{Q}\left(\Re_{n}\right)$ and $x_{n} \rightarrow x$. Further, for all $y$ in $\mathbb{Q}^{\circ}(\Re)$ and $\delta_{1}>0$, there exists $N_{1}$ such that $n>N_{1}$ implies $\left\|x_{n} y-y x_{n}\right\|<\delta_{1}$, since multiplication is continuous. We now construct a sequence $x_{n}^{\prime}, n=1,2, \ldots$, such that $x_{n}^{\prime}$ is in $\mathscr{Q}\left(\Re^{\perp}\right) \vee N(\Re)$ and $x_{n}^{\prime} \rightarrow x$ as $n \rightarrow \infty$. Let $e_{i j}^{(k)}$ be a set of matrix units for $\mathfrak{E}^{\circ}(\Re)$. Define $x_{n}^{\prime}=\sum_{i} \sum_{k} e_{i 1}^{(k)} x_{n} e_{1 i}^{(k)}, 1 \leqslant i<q<\infty, 1 \leqslant k$ $\leqslant p<\infty$. By construction $x_{n}^{\prime}$ is in $\mathscr{Q}\left(\Re_{n}\right)$ and by straightforward computation, $x_{n}^{\prime}$ is in $\mathbb{Q}^{\circ}(\Re)^{c}$. Further, 


$$
\begin{aligned}
\left\|x_{n}-x_{n}^{\prime}\right\| & =\left\|x_{n}-\sum_{i} \sum_{k} e_{i 1}^{(k)} x_{n} e_{1 i}^{(k)}\right\|=\left\|e x_{n}-\sum_{i} \sum_{k} e_{i 1}^{(k)} x_{n} e_{1 i}^{(k)}\right\| \\
& =\left\|\sum_{i} \sum_{k} e_{i i}^{(k)} x_{n}-\sum_{i} \sum_{k} e_{i 1}^{(k)} x_{n} e_{1 i}^{(k)}\right\| \leqslant \sum_{i} \sum_{k}\left\|e_{i i}^{(k)} x_{n}-e_{i 1}^{(k)} x_{n} e_{1 i}^{(k)}\right\| \\
& =\sum_{i} \sum_{k}\left\|e_{i 1}^{(k)}\left(e_{1 i}^{(k)} x_{n}-x_{n} e_{1 i}^{(k)}\right)\right\| \leqslant \sum_{i} \sum_{k}\left\|e_{1 i}^{(k)} x_{n}-x_{n} e_{1 i}^{(k)}\right\| .
\end{aligned}
$$

Thus for $n>N_{1}$, we have $\left\|x_{n}-x_{n}^{\prime}\right\| \leqslant p q \delta_{1}$. Also, since $x_{n} \rightarrow x$, for any $\delta_{2}>0$ there is an $N_{2}$ such that $n>N_{2}$ implies $\left\|x-x_{n}\right\|<\delta_{2}$. We now pick $\delta_{1}=\varepsilon / 2 p q$ and $\delta_{2}=\varepsilon / 2$, and $N=\max \left(N_{1}, N_{2}\right)$. Combining, we obtain $\left\|x-x_{n}^{\prime}\right\|<\varepsilon$. Thus $x_{n}^{\prime} \rightarrow x$ as $n \rightarrow \infty, x_{n}^{\prime}$ is in $\mathscr{Q}\left(\pi_{n}\right)$, and denoting the commutant relative to $\mathscr{Q}\left(\pi_{n}\right)$ by $c$, we have $x_{n}^{\prime}$ in $\mathbb{Q}^{\circ}(\Re)^{c}=\mathbb{Q}\left(\Re^{\perp}\right) \vee N(\Re)$ by Lemma 3.6, since $\Re \subset \Re_{n}$ and both are finite-dimensional. Finally, since $x_{n}^{\prime} \rightarrow x$ we conclude $x$ in $\mathscr{Q}\left(\Re^{\perp}\right) \vee N(\Re)$. Done.

LEMMA 3.8. Let $\Re$ and $\Re$ be finite-dimensional Hilbert spaces such that $\pi \subset \Re$. Let $c_{0}$ denote the commutant relative to $\mathbb{Q}^{\circ}(\Re)$. Then $\mathbb{Q}^{\circ}(\Re)^{c_{0}}$ $=\mathbb{Q}^{\circ}\left(\mathscr{N}^{\perp}\right) \vee z\left(\mathbb{Q}^{\circ}(\Re)\right)=\mathbb{Q}^{\circ}\left(\Re^{\perp}\right) \vee N(\Re)$.

Proof. Since $z\left(\mathscr{Q}^{\circ}(\Re)\right)=\mathscr{Q}(N(\Re))$ by Lemma 3.4, the second equality is immediate. Now we show $\mathscr{Q}^{\circ}(\Re)^{c_{0}}=\mathbb{Q}^{\circ}\left(\Re^{\perp}\right) \vee N(\Re)$. Consider $x$ in $\mathbb{Q}^{\circ}(\Re)^{c_{0}} \subset \mathbb{Q}^{\circ}(\Re)^{c}$. By Lemma 3.6 and the fact that $z\left(\mathbb{Q}^{\circ}(\Re)\right)=\mathbb{Q}(N(\Re))$, we can write $x=\sum_{i} y_{i} z_{i}$ with $y_{i}$ in $\mathscr{Q}\left(\Re^{\perp}\right)$ and $z_{i}$ in $\mathscr{Q}(N(\Re))$. Letting $\chi_{t}$ be the gauge automorphism and

$$
\alpha=\frac{1}{2 \pi} \int_{0}^{2 \pi} x_{t} d t
$$

we note that $\alpha: \mathscr{Q}(\Re) \rightarrow \mathbb{Q}^{\circ}(\Re)$ and leaves $\mathbb{Q}^{\circ}(\Re)$ fixed. Hence for $x$ in $\mathbb{Q}^{\circ}(\Re)^{c_{0}} \subset \mathbb{Q}^{\circ}(\Re)$ we have $\alpha(x)=x=\sum_{i} \alpha\left(y_{i} z_{i}\right)=\sum_{i} \alpha\left(y_{i}\right) z_{i}$, using the fact that $N(\Re)$ and thus $z_{i}$ is gauge invariant. Further, since $y_{i}$ is in $\mathbb{Q}^{\circ}\left(\Re^{\perp}\right), \alpha\left(y_{i}\right)$ is in $\mathbb{Q}^{\circ}\left(\pi^{\perp}\right)$, and we conclude $\mathbb{Q}^{\circ}(\Re)^{c_{0}}=\mathbb{Q}^{\circ}\left(\pi^{\perp}\right)$ $\checkmark N(\Re)$. Done.

Lemma 3.9. Let $\nVdash$ be a separable Hilbert space, and $\pi \subset \Re$ a finitedimensional subspace. Let $c_{0}$ denote the commutant relative to $\mathbb{Q}^{\circ}(\mathcal{K})$. Then $\mathbb{Q}^{\circ}(\Re)^{c_{0}}=\mathbb{Q}^{\circ}\left(\Re^{\perp}\right) \vee z\left(\mathbb{Q}^{\circ}(\Re)\right)$.

Proof. (つ) Trivially, $z\left(\mathscr{Q}^{\circ}(\Re)\right) \subset \mathbb{Q}^{\circ}(\Re)^{c_{0}}$, so we need only show $\mathbb{Q}^{\circ}\left(\mathscr{N}^{\perp}\right) \subset \mathbb{Q}^{\circ}(\Re)^{c_{0}}$. Certainly $\mathbb{Q}^{\circ}\left(\Re^{\perp}\right) \subset \mathbb{Q}^{\circ}(\mathscr{K})$, and $\mathbb{Q}^{\circ}\left(\Re^{\perp}\right) \subset \mathbb{Q}\left(\Re^{\perp}\right)$ $\subset \mathbb{Q}^{\circ}(\Re)^{c}$. Hence $\mathbb{Q}^{\circ}\left(\mathscr{N}^{\perp}\right) \subset \mathbb{Q}^{\circ}(\Re)^{c_{0}}$. Done.

(C) Let $x$ be in $\mathscr{Q}^{\circ}(\Re)^{c_{0}}$, and let $\left\{\Re_{k}\right\}, k=1,2, \ldots$, be an increasing sequence of subspaces of $\nVdash$ such that $\Re=\Re_{1}$ and $\overline{U_{n} \Re_{n}}=\nVdash$. Certainly, $x$ is in $\mathcal{Q}^{\circ}(\mathcal{K})=\overline{U_{n} \mathbb{Q}^{\circ}\left(\Re_{n}\right)}$, hence there exists a sequence $\left\{x_{n}\right\}, n=1,2, \ldots$, 
such that $x_{n}$ is in $\mathbb{Q}^{\circ}\left(\Re_{n}\right)$ and $x_{n} \rightarrow x$. Arguing exactly as in the same inclusion of Lemma 3.7, we may construct a sequence $\left\{x_{n}^{\prime}\right\}, n=1,2, \ldots$, such that $x_{n}^{\prime}$ is in $\mathbb{Q}^{\circ}\left(\mathscr{N}^{\perp}\right) \vee z\left(\mathbb{Q}^{\circ}(\Re)\right)$ and $x_{n}^{\prime} \rightarrow x$ as $n \rightarrow \infty$, using Lemma 3.8 to conclude $x_{n}^{\prime}$ is in $\mathbb{Q}^{\circ}\left(\Re^{\perp}\right) \vee z\left(\mathbb{Q}^{\circ}(\Re)\right)$ for each $n=1,2, \ldots$ Done.

LEMMA 3.10. Let $\mathbb{Q}^{\circ}(\mathscr{K})=\overline{U_{r} \mathbb{Q}^{\circ}\left(\Re_{r}\right)}$, with $\left\{\Re_{r}\right\}, r=1,2, \ldots$, an increasing sequence of r-dimensional subspaces of $\Re$ such that $\bar{U}_{r} \pi_{r}=\Re$. For convenience, let $N_{r}=N\left(\Re_{r}\right)$. Then $z\left(\mathbb{Q}^{\circ}\left(\Re_{r}\right)\right)=\mathbb{Q}\left(N_{r}\right)$ is spanned by $r+1$ orthogonal projections $E_{0}, \ldots, E_{r}$.

Proof. By a straightforward analysis of the matrix units [4, Lemma 5.2] we have $\mathbb{Q}^{\circ}\left(\mathfrak{T}_{r}\right)$ isomorphic to a direct sum of $r+1$ finite-dimensional factors. Defining $E_{k}$ to be the projection on the $k$ th such factor, $0<k<r$, we have $r+1$ orthogonal projections which clearly $\operatorname{span} z\left(\mathscr{Q}^{\circ}\left(\mathscr{N}_{r}\right)\right)$. Done.

Proposition 3.11. Let $\mathbb{Q}^{\circ}(\mathscr{K})=\overline{U_{r} \mathbb{Q}^{\circ}\left(\Re_{r}\right)}$ with $\left\{\Re_{r}\right\}, r=1,2, \ldots$, an increasing sequence of r-dimensional subspaces of $\Re$ such that $\bar{U}_{r} \pi_{r}=\Re$, and $N_{r}=N\left(\pi_{r}\right)$ as above. Let $c_{0}$ denote the commutant relative to $\mathbb{Q}^{\circ}(\mathcal{H})$. Then for arbitrary $y$ in $\mathbb{Q}^{\circ}\left(\Re_{r}\right)^{c_{0}}$ we can write

$$
y=\int_{0}^{2 \pi} y(t) e^{i N_{r} t} d t
$$

with $y(t)$ in $\mathbb{Q}^{\circ}\left(\Re_{r}^{\frac{1}{}}\right)$.

PROOF. Let $y$ be in $\mathbb{Q}^{\circ}\left(\pi_{r}\right)^{c_{0}}$. By Lemmas 3.9 and 3.4 we have $\mathbb{Q}^{\circ}\left(\pi_{r}\right)^{c_{0}}$ $=\mathbb{Q}^{\circ}\left(\pi_{r}^{\perp}\right) \vee N_{r}$; thus $N_{r}$ in $\mathbb{Q}^{\circ}\left(\Re_{r}\right)$ commutes with $\mathbb{Q}^{\circ}\left(\pi_{r}^{\perp}\right)$. Using Lemma 3.10 , then, we can write $y=\sum_{k=0}^{r} y_{k} E_{k}$ with $y_{k}$ in $\mathscr{Q}^{\circ}\left(\Re_{r}^{+}\right)$. Now letting

$$
y(t)=\frac{1}{2 \pi} \sum_{k=0}^{r} y_{k} e^{-i k t}
$$

we have $y(t)$ in $\mathbb{Q}^{\circ}\left(\pi_{r}^{+}\right)$since the $y_{k}$ are. Now

$$
\begin{aligned}
e^{i N_{r} t} & =\exp \left(i \sum_{j=0}^{r} a\left(f_{j}\right)^{*} a\left(f_{j}\right) t\right)=\prod_{j=0}^{r} \exp \left(i e_{22}^{(j)} t\right) \\
& =\prod_{j=0}^{r}\left(e_{11}^{(j)}+e_{22}^{(j)} e^{i t}\right)
\end{aligned}
$$

and we obtain

$$
\int_{0}^{2 \pi} y(t) e^{i N_{r} t} d t=\frac{1}{2 \pi} \sum_{k=0}^{r} y_{k} \int_{0}^{2 \pi} e^{-i k t} \times \prod_{j=0}^{r}\left(e_{11}^{(j)}+e_{22}^{(j)} e^{i t}\right) d t .
$$

Clearly, this integral picks out of the product the coefficient of the $e^{i k t}$ term. An inspection of the matrix units for $\mathbb{Q}^{\circ}\left(\pi_{r}\right)$ shows that these coefficients are 
the central projections $E_{k}$ of $\mathfrak{Q}^{\circ}\left(\Re_{r}\right)$ above. Hence we have

$$
\int_{0}^{2 \pi} y(t) e^{i N, t} d t=\sum_{k=0}^{r} y_{k} E_{k}=y .
$$

Done.

In the following section, we will relate the factor condition for a given generalized free state to properties of a particular series. We begin with some definitions.

DefinItion 3.12. Given a function $f(t)=\sum_{r=0}^{\infty} \gamma_{r} e^{i r t}$, we define its Fourier$s$ norm, denoted \|\|$_{s}$, by the formula

$$
\|f\|_{s}=\left\{\sum_{r=0}^{\infty}\left|\gamma_{r}\right|^{s}\right\}^{1 / s}
$$

DefinITION 3.13. Let $\omega$ be a state of $\mathscr{Q}^{\circ}(\mathcal{K})=\overline{U_{r} \mathbb{Q}^{\circ}\left(\mathscr{T}_{r}\right)}$ with $\left\{\mathscr{N}_{r}\right\}, r=1$, $2, \ldots$, an increasing sequence of finite-dimensional subspaces of $\mathcal{H}$ such that $\bar{U}_{r} \Re_{r}=\Re_{\text {. Let }} N_{r}=N\left(\Re_{r}\right)$ as above. We define a function $P_{m n}(t)$ associated with $\omega$ by the formula $P_{m n}(t)=\omega\left(e^{i\left(N_{m}-N_{n}\right) t}\right)$.

Proposition 3.14. Let $\omega$ be a generalized free state of $\mathscr{Q}(\mathcal{K})=\overline{U_{r} \mathscr{Q}\left(\Re_{r}\right)}$, which factorizes with respect to the increasing sequence of finite-dimensional subspaces $\left\{\Re_{r}\right\}, r=1,2, \ldots$ Let $x$ be in $\mathbb{Q}^{\circ}\left(\Re_{n}\right)$, and $y$ be in $\mathbb{Q}^{\circ}\left(\pi_{m}\right)^{c_{0}}, m$ $>n$. Then $|\omega(x y)-\omega(x) \omega(y)| \rightarrow 0$ as $m \rightarrow \infty$ if $\left\|\left(1-e^{i t}\right) P_{m n}(t)\right\|_{1} \rightarrow 0$ as $m \rightarrow \infty$.

Proof. By Lemma 3.11 and the continuity of $\omega$, we have

$$
\omega(x y)-\omega(x) \omega(y)=\int_{0}^{2 \pi}\left(\omega\left(x y(t) e^{i N_{m} t}\right)-\omega(x) \omega\left(y(t) e^{i N_{m} t}\right)\right) d t .
$$

Also,

$$
\omega\left(x y(t) e^{i N_{m} t}\right)=\omega\left(x e^{i N_{m} t} y(t)\right)=\omega\left(x e^{i N_{m} t}\right) \omega(y(t))
$$

and

$$
\omega\left(y(t) e^{i N_{m} t}\right)=\omega(y(t)) \omega\left(e^{i N_{m} t}\right)
$$

using the factorization properties of $\omega$. Now noting $e^{i N_{n} t}=e^{i\left(N_{m}-N_{n}\right) t} e^{i N_{n} t}$ and combining, we obtain

$$
\begin{aligned}
\omega(x y)-\omega(x) \omega(y) & =\int_{0}^{2 \pi} \omega(y(t)) P_{m n}(t)\left[\omega\left(x e^{i N_{n} t}\right)-\omega(x) \omega\left(e^{i N_{n} t}\right)\right] d t \\
& =\int_{0}^{2 \pi} \omega(y(t)) P_{m n}(t) q_{x}(t) d t
\end{aligned}
$$


defining $q_{x}(t)=\omega\left(x e^{i N_{n} t}\right)-\omega(x) \omega\left(e^{i N_{n} t}\right)$. Now $q_{x}(0)=0$ and, recalling $E_{k}, k$ $=0,1, \ldots, n$, to be the central projections of $\mathbb{Q}^{\circ}\left(\mathscr{N}_{n}\right)$ we have $e^{i N_{n} t}$ $=\sum_{k=0}^{n} E_{k} e^{i k t}$ by straightforward comparison. Substituting, we see that $q_{x}(t)$ is a finite Fourier sum with highest power $n$. Hence, there are coefficients $a_{k}, k=1,2, \ldots, n$, such that

$$
q_{x}(t)=\sum_{k=1}^{n} a_{k}\left(1-e^{i k t}\right) .
$$

Further, $y(t)=(1 / 2 \pi) \sum_{p=0}^{m} y_{p} e^{-i p t}$, and defining $\omega\left(y_{p}\right)=b_{p}$, we get $\omega(y(t))$ $=(1 / 2 \pi) \sum_{p=0}^{m} b_{p} e^{-i p t}$. Substituting these into our last expression for $\omega(x) \omega(y)$, we obtain

$$
\begin{aligned}
\omega(x y)-\omega(x) \omega(y) & =\int_{0}^{2 \pi}\left(\sum_{p=0}^{m} b_{p} e^{-i p t}\right)\left(\sum_{k=1}^{n} a_{k}\left(1-e^{i k t}\right)\right) P_{m n}(t) d t \\
& =\sum_{k=1}^{n} \sum_{p=0}^{m} a_{k} b_{p} \int_{0}^{2 \pi} e^{-i p t}\left(1-e^{i k t}\right) P_{m n}(t) d t .
\end{aligned}
$$

Now define $\sum_{r=0}^{m-n+k} c_{r} e^{i r t}=\left(1-e^{i k t}\right) P_{m n}(t)$; the sum limit follows from the equation

$$
e^{i\left(N_{m}-N_{n}\right) t}=\prod_{l=n}^{m}\left(e_{11}^{(l)}+e_{22}^{(l)} e^{i t}\right) .
$$

Substituting and taking absolute values, we get

$$
\begin{aligned}
|\omega(x y)-\omega(x) \omega(y)| & =\left|\sum_{k=1}^{n} \sum_{p=0}^{m} \sum_{r=0}^{m-n+k} a_{k} b_{p} c_{r} \int_{0}^{2 \pi} e^{-i p t} e^{i r t} d t\right| \\
& =\left|2 \pi \sum_{k=1}^{n} \sum_{r=0}^{m-n+k} a_{k} b_{r} c_{r}\right| \\
& \leqslant 2 \pi \sum_{k=1}^{n}\left|a_{k}\right| \sum_{r=0}^{m-n+k}\left|b_{r}\right|\left|c_{r}\right| \leqslant 2 \pi \sum_{k=1}^{n}\left|a_{k}\right| \sum_{r=0}^{m-n+k}\left|c_{r}\right|
\end{aligned}
$$

since we may assume $\|y\|=1$, and it follows that $\left|b_{r}\right| \leqslant 1$. Now note $n$ is fixed and $k \leqslant n$, so $m \rightarrow \infty$ implies $m-n+k \rightarrow \infty$. Hence, for arbitrary $x$ in $\mathbb{Q}^{\circ}\left(\pi_{n}\right)$ and $y$ in $\mathbb{Q}^{\circ}\left(\pi_{m}\right)^{c_{0}}$ we have $|\omega(x y)-\omega(x) \omega(y)| \rightarrow 0$ as $m \rightarrow \infty$ if $\sum_{r=0}^{m-n+k}\left|c_{r}\right| \rightarrow 0$ as $m \rightarrow \infty$. By definition, $\sum_{r=0}^{m-n+k}\left|c_{r}\right|=\left\|\left(1-e^{i k t}\right) P_{m n}(t)\right\|_{1}$, and observing $\left(1-e^{i k t}\right)=\sum_{s=0}^{k-1} e^{i s t}\left(1-e^{i t}\right)$ we obtain

$$
\begin{aligned}
\left\|\left(1-e^{i k t}\right) P_{m n}(t)\right\|_{1} & =\left\|\sum_{s=0}^{k-1} e^{i s t}\left(1-e^{i t}\right) P_{m n}(t)\right\|_{1} \\
& \leqslant(n-1)\left\|\left(1-e^{i t}\right) P_{m n}(t)\right\|_{1}
\end{aligned}
$$


since $k \leqslant n$. But $n$ is fixed, so $\left\|\left(1-e^{i t}\right) P_{m n}(t)\right\|_{\mathfrak{l}} \rightarrow 0$ as $m \rightarrow \infty$ implies $|\omega(x y)-\omega(x) \omega(y)| \rightarrow 0$ as $m \rightarrow \infty$. Done.

LEMMA 3.15. Let $A$ be a positive operator on a Hilbert space $\Re_{\text {, with }}$ $0<A<I$, pure point spectrum $\left\{\lambda_{i}\right\}, i=1,2, \ldots$, and associated eigenvectors $\left\{f_{i}\right\}, i=1,2, \ldots$ Let $\Re_{r}=\operatorname{span}\left\{f_{1}, \ldots, f_{r}\right\}$ and let $\omega_{A}$ be the gauge invariant

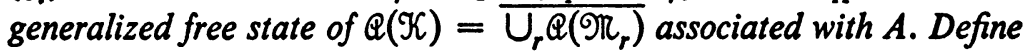

$$
P_{m n}(t)=\omega_{A}\left(e^{i\left(N_{m}-N_{n}\right) t}\right)
$$

as above. Then

$$
\left\|\left(1-e^{i t}\right) P_{m n}(t)\right\|_{1}=\left\|\left(1-e^{i t}\right) \prod_{j=n+1}^{m}\left\{\left(1-\lambda_{j}\right)+\lambda_{j} e^{i t}\right\}\right\|_{1} .
$$

Proof. Recall

$$
P_{m n}(t)=\omega_{A}\left(e^{i\left(N_{m}-N_{n} t\right.}\right)=\omega_{A}\left(\prod_{j=n+1}^{m} e_{11}^{(j)}+e^{i t} e_{22}^{(j)}\right) .
$$

By construction, $\omega_{A}$ factorizes with respect to the $\left\{\pi_{r}\right\}, r=1,2, \ldots$, and the $e_{i j}^{(k)}$ lie in $\mathfrak{B}_{k}$ with

$$
\mathfrak{Q}(\mathscr{K}) \approx \bigotimes_{k=1}^{\infty} \mathfrak{Q}\left(\mathscr{R}_{k+1}\right) \cap \mathfrak{Q}\left(\mathscr{T}_{k}\right)^{c}=\bigotimes_{k=1}^{\infty} \mathscr{B}_{k} .
$$

Hence

$$
\begin{aligned}
P_{m n}(t) & =\prod_{j=n+1}^{m}\left\{\omega_{A}\left(e_{11}^{(j)}\right)+e^{i t} \omega_{A}\left(e_{22}^{(j)}\right)\right\} \\
& =\prod_{j=n+1}^{m}\left\{\left(1-\lambda_{j}\right)+\lambda_{j} e^{i t}\right\} . \quad \text { Done. }
\end{aligned}
$$

Definition 3.16. We define the functions $P_{n}$ and $\chi_{n}$ by the formulae

$$
\begin{aligned}
P_{n}(t) & =\prod_{j=1}^{n}\left\{\left(1-\lambda_{j}\right)+\lambda_{j} e^{i t}\right\}, \\
\chi_{n}(t) & =\left(1-e^{i t}\right) P_{n}(t) .
\end{aligned}
$$

Definition 3.17. We define the Fourier coefficients $\alpha_{k}$ and $\gamma_{k}$ by the formulae

$$
P_{n}(t)=\sum_{k=0}^{n} \alpha_{k} e^{i k t}, \quad \chi_{n}(t)=\sum_{k=0}^{n+1} \gamma_{k} e^{i k t} .
$$

REMARK. Since $\chi_{n}(t)=\left(1-e^{i t}\right) P_{n}(t)$, we observe 


$$
\gamma_{k}=\alpha_{k}-\alpha_{k-1} \text { for } 0 \leqslant k \leqslant n+1 \text { taking } \alpha_{-1}=\alpha_{n+1}=0
$$

LEMMA 3.18 (CHANGE OF SIGN). Let $\chi_{n}(t)=\sum_{k=0}^{n+1} \gamma_{k} e^{i k t}$ as above. Then, for all $n>0$, there is a $p<n$ such that

$$
\gamma_{k} \begin{cases}\geqslant 0, & k \leqslant p \\ \leqslant 0, & k>p .\end{cases}
$$

Proof. By induction. For $n=1$, we have

$$
\chi_{1}=\left(1-e^{i t}\right)\left\{\left(1-\lambda_{1}\right)+\lambda_{1} e^{i t}\right\}
$$

whose coefficients are $1-\lambda_{1}, 2 \lambda_{1}-1,-\lambda_{1}$, in order, and clearly

$$
p= \begin{cases}1, & \lambda_{1}<\frac{1}{2}, \\ 2, & \lambda_{1} \geqslant \frac{1}{2} .\end{cases}
$$

Now suppose true for $\chi_{n}$. We can write the coefficients in order as $a_{n_{+}}, \ldots$, $a_{3}, a_{2}, a_{1},-b_{1},-b_{2},-b_{3}, \ldots,-b_{n_{-}}$with $n_{+}+n_{-}=n+2, p=n_{+}$, and $a_{i}$, $b_{i} \geqslant 0$. Since $x_{n+1}=\left(\left(1-\lambda_{n+1}\right)+\lambda_{n+1} e^{i t}\right) x_{n}$ we can generate the $x_{n+1}$ coefficients as indicated below:

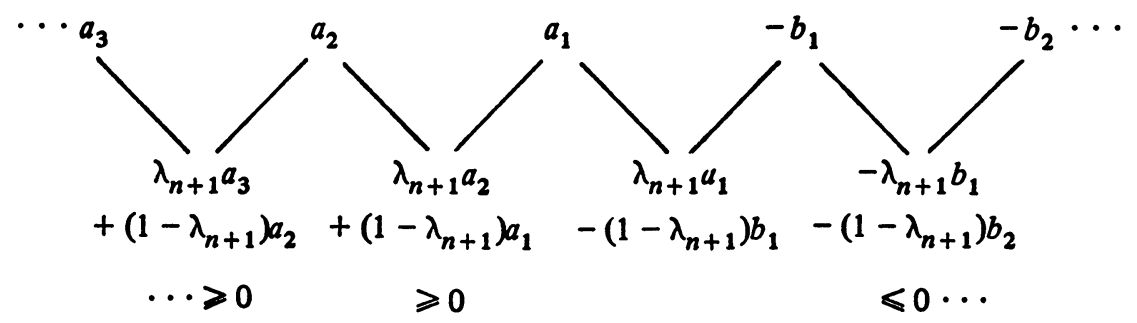

FIGURE 1. Generation of coefficients

Hence we have the two cases

$$
\lambda_{n+1} a_{1}-\left(1-\lambda_{n+1}\right) b_{1}\left\{\begin{array}{l}
\geqslant 0 \\
<0,
\end{array}\right.
$$

and we conclude

$$
p=\left\{\begin{array}{l}
n_{+}+1 \\
n_{+},
\end{array}\right.
$$

respectively.

Proposition 3.19. Let $\chi_{n}(t), P_{n}(t)$ and $\alpha_{k}$ be as in Definitions 3.16 and 3.17. Then 


$$
\left\|x_{n}\right\|_{1}=2 \max _{0<k<n}\left\{\alpha_{k}\right\}
$$

Proof. Recall

$$
P_{n}(t)=\sum_{k=0}^{n} \alpha_{k} e^{i k t}, \quad \chi_{n}(t)=\sum_{k=0}^{n+1} \gamma_{k} e^{i k t}, \gamma_{k}=\alpha_{k}-\alpha_{k-1},
$$

with $\alpha_{k}=0$ for $k<0$ or $k>n$. By the change of sign lemma (Lemma 3.18) there is a $p$ such that

$$
\begin{array}{ll}
\gamma_{k} \geqslant 0, & k=0,1, \ldots, p-1, \\
\gamma_{k} \leqslant 0, & k=p, p+1, \ldots, n+1 .
\end{array}
$$

Thus,

$$
\begin{aligned}
\left\|\chi_{n}\right\|_{1} & =\sum_{k=0}^{n+1}\left|\gamma_{k}\right|=\sum_{k=0}^{p-1} \gamma_{k}-\sum_{k=p}^{n+1} \gamma_{k} \\
& =\sum_{k=0}^{p-1}\left(\alpha_{k}-\alpha_{k-1}\right)-\sum_{k=p}^{n+1}\left(\alpha_{k}-\alpha_{k-1}\right) \\
& =\left\{\alpha_{0}+\left(\alpha_{1}-\alpha_{0}\right)+\cdots+\left(\alpha_{p-1}-\alpha_{p-2}\right)\right\} \\
& -\left\{\left(\alpha_{p}-\alpha_{p-1}\right)+\left(\alpha_{p+1}-\alpha_{p}\right)+\cdots+\left(\alpha_{n}-\alpha_{n-1}\right)-\alpha_{n}\right\} \\
& =\alpha_{p-1}-\left(-\alpha_{p-1}\right)=2 \alpha_{p-1} .
\end{aligned}
$$

But $\alpha_{p-1}=\max _{0<k<n}\left\{\alpha_{k}\right\}$. Hence

$$
\left\|\chi_{n}\right\|_{1}=2 \alpha_{p-1}=2 \max _{0<k<n}\left\{\alpha_{k}\right\} .
$$

THEOREM 3.20. Let $A$ be a positive operator on a separable Hilbert space K with $0<A<I$ and pure point spectrum $\left\{\lambda_{i}\right\}, i=1,2, \ldots$. Then $\omega_{A}^{\circ}$, the associated generalized free state of $\mathbb{Q}^{\circ}(\mathcal{K})$, is a factor state if $\sum_{i=1}^{\infty} \lambda_{i}\left(1-\lambda_{i}\right)=\infty$.

Proor. By Proposition 3.14, given $\left\|\left(1-e^{i t}\right) P_{m n}(t)\right\|_{1} \rightarrow 0$ as $m \rightarrow \infty$, we have $\left|\omega_{A}^{\circ}(x y)-\omega_{A}^{\circ}(x) \omega_{A}^{\circ}(y)\right| \rightarrow 0$ as $m \rightarrow \infty$ for $x$ in $\mathbb{Q}^{\circ}\left(\Re_{n}\right), y$ in $\mathbb{Q}^{\circ}\left(\mathscr{N}_{m}\right)^{c_{0}}$. Since $\omega_{A}^{\circ}$ is continuous and the $\cup_{n} \mathbb{Q}^{\circ}\left(\Re_{n}\right)$ is dense in $\mathscr{Q}^{\circ}(\mathscr{K})$ we may assume $x$ is an arbitrary element of $\mathscr{Q}^{\circ}(\mathcal{H})$. Now invoking Theorem 3.1, Lemma 3.15 and Definition 3.16 (relabeling the product lower limit $n+1$ ) we conclude $\omega_{A}^{\circ}$ is a factor state if $\left\|\chi_{m}\right\|_{h} \rightarrow 0$ as $m \rightarrow \infty$. By Proposition 3.19 it is sufficient to show that $\max _{0<k<m}\left\{\alpha_{k}\right\} \rightarrow 0$ as $m \rightarrow \infty$. Now note 


$$
\begin{aligned}
\left|\alpha_{k}\right| & =\left|\frac{1}{2 \pi} \int_{0}^{2 \pi} P_{m n}(t) e^{-i k t} d t\right|=\left|\int_{0}^{2 \pi} \frac{e^{i k t}}{(2 \pi)^{1 / 2}} \frac{P_{m n}}{(2 \pi)^{1 / 2}}(t) d t\right| \\
& \leqslant \frac{1}{2 \pi} \int_{0}^{2 \pi}\left|P_{m n}(t)\right|^{2} d t
\end{aligned}
$$

by the Cauchy-Schwarz inequality. Further,

$$
\left|P_{m n}(t)\right|^{2}=P_{m n}(t) P_{m n}^{*}(t)=\prod_{i=n+1}^{m}\left\{1-2(1-\cos t) \lambda_{i}\left(1-\lambda_{i}\right)\right\} .
$$

But, for all $t$ in $(0,2 \pi)$ and $n=1,2, \ldots$,

$$
\sum_{i=n+1}^{\infty} 2(1-\cos t) \lambda_{i}\left(1-\lambda_{i}\right)=2(1-\cos t) \sum_{i=n+1}^{\infty} \lambda_{i}\left(1-\lambda_{i}\right)=\infty
$$

if and only if $\sum_{i=1}^{\infty} \lambda_{i}\left(1-\lambda_{i}\right)=\infty$. Hence $\left|P_{m n}(t)\right|^{2} \rightarrow 0$ as $n \rightarrow \infty$ by the residual theorem for infinite products, for all $t$ in $(0,2 \pi)$. Finally, $\left|P_{m n}(t)\right|^{2} \leqslant 1$ and $\left|P_{m n}(t)\right| \rightarrow 0$ as $n \rightarrow \infty$ almost everywhere on $(0,2 \pi)$ so $\int_{0}^{2 \pi n}\left|P_{m n}(t)\right|^{2} d t$ $\rightarrow 0$ as $n \rightarrow \infty$. Thus $\left|\alpha_{k}\right| \rightarrow 0$ as $n \rightarrow \infty$, independent of $k$, by our first inequality. Done.

The following lemma, as modified in Proposition 3.22, will be useful in proving the main result (Theorem 3.24).

LEMMA 3.21 [LEMMA 5.3,(i)]. Let $\omega_{A}$ be a gauge invariant generalized free state of the CAR algebra $\mathbb{Q}(\mathcal{T})$. Then $\omega_{A}$ is type I if and only if there exists a spectral projection $E$ of $A$ and a trace class operator $T$ such that $A=E+T$.

Proposition 3.22. Let $A$ be a positive operator on a separable Hilbert space $\mathfrak{K}$ with $0<A<I$, and let $\omega_{A}$ be the associated generalized free state of $Q(\mathcal{F})$. Then $\omega_{A}$ induces a type I factor representation of $\mathbb{Q}(\mathcal{K})$ if and only if $\operatorname{Tr} A(I-A)$ $<\infty$.

Proor. Sufficiency. Suppose $\omega_{A}$ induces a type I factor representation. By the above lemma, $A=E+T$, with $E$ a projection and $T$ trace class. Hence

$$
A(I-A)=(E+T)((I-E)-T)=-E T+T(I-E)-T^{2}
$$

and

$$
\operatorname{Tr} A(I-A)=-\operatorname{Tr}(E T)+\operatorname{Tr}(T(I-E))-\operatorname{Tr}\left(T^{2}\right)<\infty
$$

since the trace class operators form an ideal in $\mathscr{B}(\mathcal{K})$.

Necessity. Suppose $\operatorname{Tr} A(I-A)<\infty$. Then $A$ clearly has pure point spectrum, and there is an orthonormal basis $\left\{f_{i}\right\}, i=1,2, \ldots$, for $\nVdash$ such that $A f_{i}=\lambda_{i} f_{i}$ and $0 \leqslant \lambda_{i} \leqslant 1$. Let $E$ be the spectral projection of $A$ projecting onto $\frac{\lambda_{i}}{\operatorname{span}}\left(\left\{f_{i}: \lambda_{i} \geqslant \frac{1}{2}\right\}\right)$. Then $A E \geqslant \frac{1}{2} E$ and 


$$
\operatorname{Tr}(|A-E|)=\sum_{i}\left(f_{i},(|A-E|) f_{i}\right)=\sum_{i \in I-E} \lambda_{i}+\sum_{i \in E}\left(1-\lambda_{i}\right)
$$

where $i \in E$ means $A f_{i}=\lambda_{i} f_{i}$ and $\lambda_{i} \geqslant \frac{1}{2}, i \in(I-E)$ means $A f_{i}=\lambda_{i} f_{i}$ and $\lambda_{i}<\frac{1}{2}$. Note that

$$
\lambda_{i}\left(1-\lambda_{i}\right) \leqslant \min \left(\lambda_{i}, 1-\lambda_{i}\right) \leqslant 2 \lambda_{i}\left(1-\lambda_{i}\right)
$$

follows straightforwardly from $0<\lambda_{i}<1$. Hence

$$
\sum_{i=1}^{\infty} \lambda_{i}\left(1-\lambda_{i}\right) \leqslant \sum_{i \in I-E} \lambda_{i}+\sum_{i \in E}\left(1-\lambda_{i}\right) \leqslant 2 \sum_{i=1}^{\infty} \lambda_{i}\left(1-\lambda_{i}\right)
$$

or $\operatorname{Tr}(A-E)<\infty$ if and only if $\sum_{i=1}^{\infty} \lambda_{i}\left(1-\lambda_{i}\right)<\infty$. Thus $A-E$ is trace class or $A=E+T$ with $T$ trace class, which implies $\omega_{A}$ induces a type I factor representation by the above lemma. Done.

In proving the main result of this section (Theorem 3.24) we will (a) make use of [14, Theorem 1] where sufficiency (i.e., $\omega_{A}^{\circ}$ is a factor state implies $\operatorname{Tr} A(I-A)=\infty)$ is already proved and (b) invoke Theorem 4.12 and Lemmas 3.23, 4.10, 4.16 for the necessity argument. We now state

LEMma 3.23 [12, Lemma 2.1]. Let $\Re$ be a Hilbert space and $\omega_{1}, \omega_{2}$ two factor states of $\mathscr{Q}(\mathcal{H})$. Let $\Re_{1} \subset \mathbb{R}_{2} \cdots$ be an increasing sequence of finite-dimension-

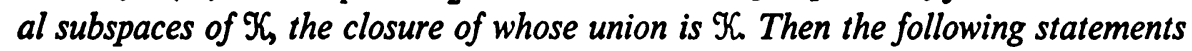
are equivalent:

(i) $\omega_{1} \tau_{q} \omega_{2}$.

(ii) For every $\varepsilon>0$ there is an integer $n$ such that

$$
\left\|\omega_{1}\left|\mathscr{Q}\left(\Re_{n}\right)^{c}-\omega_{2}\right| \mathscr{Q}\left(\Re_{n}\right)^{c}\right\|<\varepsilon .
$$

(iii) There is a finite-dimensional subspace $\Re \subset \Re$ such that

$$
\left\|\omega_{1}\left|\mathscr{Q}(\Re)^{c}-\omega_{2}\right| \mathscr{Q}(\Re)^{c}\right\|<2 .
$$

Finally, we have

THEOREM 3.24. Let $A$ be a positive operator on a separable Hilbert space $\Re_{\text {, }}$ with $0<A<I$. Suppose $A$ is not a projection, and let $\omega_{A}^{\circ}$ be the associated generalized free state of $\mathbb{Q}^{\circ}(\mathcal{F})$. Then $\omega_{A}^{\circ}$ is a factor state if and only if $\operatorname{Tr} A(I-A)=\infty$.

Proor. Sufficiency. This is [14, Theorem 1]. The proof proceeds by assuming $\operatorname{Tr} A(I-A)<\infty$, and constructing nontrivial central elements of the von Neumann algebra associated with the representation. These are given by $\exp \left(i \Pi\left(V_{n}\right) t\right)$ where $V_{n}=N_{n}-\omega_{A}\left(N_{n}\right) I, 0<t<1$. We note that this construction is repeated, with minor modification, in [3] to which we refer the reader for further detail. 
Necessity. Suppose $\operatorname{Tr} A(I-A)=\infty$. By Lemma 4.16 we can get a sequence of operators $\left\{A_{p}\right\}, p=1,2, \ldots$, such that

(i) $0<A_{p}<I$,

(ii) $A_{p}$ has pure point spectrum,

(iii) $\left\|A^{1 / 2}-A_{p}^{1 / 2}\right\|_{\text {H.S. }} \rightarrow 0$ as $p \rightarrow \infty$,

(iv) $\left\|(I-A)^{1 / 2}-\left(I-A_{p}\right)^{1 / 2}\right\|_{\mathrm{H} . S .} \rightarrow 0$ as $p \rightarrow \infty$.

Further, by Lemma 4.10 we have

(v) $\left\|\omega_{A}-\omega_{A_{p}}\right\| \rightarrow 0$ as $p \rightarrow \infty$,

with $\omega_{A}, \omega_{A_{p}}$ the gauge invariant generalized free states of $\mathbb{Q}(\mathcal{K})$ associated with $A, A_{p}$. Now, by Lemma 3.23 we have $\omega_{A} \tau_{q} \omega_{A_{p}}, p=1,2, \ldots$ But $\operatorname{Tr} A(I-A)=\infty$ implies $\omega_{A}$ induces a factor representation which is not type I; since $\omega_{A} \sim_{q} \omega_{A_{p}}$ we have $\omega_{A_{p}}$ inducing a factor representation not of type I. (Recall quasi-equivalent states induce representations whose associated von Neumann algebras are *-isomorphic, and type is an isomorphism invariant.) Since $\omega_{A_{p}}$ is not type I, we have $\operatorname{Tr} A_{p}\left(I-A_{p}\right)=\infty$ by Proposition 3.22. Since the $A_{p}$ have pure point spectrum, we conclude the $\omega_{A_{p}}^{\circ}$ are factor states of $\mathbb{Q}^{\circ}(\mathcal{K})$ by Theorem 3.20. But $\left\|\omega_{A}^{\circ}-\omega_{A_{p}}^{\circ}\right\| \leqslant\left\|\omega_{A}-\omega_{A_{p}}\right\| \rightarrow 0$ as $p \rightarrow \infty$ and the uniform limit of factor states is a factor state; hence $\omega_{A}^{\circ}$ is a factor state. Done.

We conclude this section by proving that the representations induced by such factor states of $\mathbb{Q}^{\circ}(\mathcal{K})$ are faithful, i.e., they have trivial kernel-hence no nontrivial two-sided ideals are mapped to zero. To do so, we use the characterization of all two-sided ideals of $\mathbb{Q}^{\circ}(\mathcal{K})$ given in $[4,5.5]$. We summarize this information with a remark.

REMARK. The ideals of $\mathscr{Q}^{\circ}(\mathcal{K})$, denoted ${ }_{n} I_{m}$ :

(i) are generated by the factors $M_{(n+m, n)}$,

(ii) contain as a dense set all gauge invariant polynomials in $a(f), a(g)^{*}$ each of whose addends has:

(a) at least $m$ creators when ordered so that all creators are standing to the left of all annihilators and

(b) at least $n$ annihilators when ordered so that all annihilators are standing to the left of all creators.

We are now ready for

Proposition 3.25. Let $A$ be a positive operator on a separable Hilbert space $\Re$ such that $0<A<I$ and $\operatorname{Tr} A(I-A)=\infty$. Let $\omega_{A}^{\circ}$ be the associated generalized free factor state of $\mathscr{Q}^{\circ}(\Re)$, and $\Pi_{A}$ the representation of $\mathbb{Q}^{\circ}(\mathcal{K})$ induced by $\omega_{A}^{\circ}$ via the Gel'fand-Naimark-Segal construction. Then $\Pi_{A}$ is faithful.

Proor. First define $\Re_{0,1}=\{f: A f=0$ or $A f=f\}$. Clearly, $\Re_{\Theta} \Theta K_{0,1}$ is infinite dimensional, since if not, $\operatorname{Tr} A(I-A)<\infty$. Now assume $\Pi_{A}$ is not faithful; if so it must map one of the ${ }_{n} I_{m}$ to zero. We observe that, by (ii) 
above, ${ }_{n} I_{m} \subset{ }_{p} I_{p}$ with $p=\max (m, n)$ and by (i), $M_{(2 p, p)}$ generates ${ }_{p} I_{p}$. Further, the element $y=e_{11}^{(1)} \cdots e_{11}^{(p)} \cdot e_{22}^{(p+1)} \cdots e_{22}^{(2 p)}$ is in $M_{(2 p, p)}$ by its definition; hence it is in ${ }_{p} I_{p}$. We now construct an element $x$ in ${ }_{n} I_{m}$ such that $\Pi_{A}(x) \neq 0$. To do so, we observe that: (a) we can find $2 p$ orthonormal vectors $\left\{g_{1}, \ldots, g_{2 p}\right\}$ in $\nVdash \ominus \mathcal{K}_{0,1}$ since it is infinite dimensional; (b) $A$ is selfadjoint when restricted to $\Re=\operatorname{span}\left(\left\{g_{1}, \ldots, g_{2 p}\right\}\right)$; (c) therefore we can find $\left\{f_{1}, \ldots, f_{2 p}\right\}$ which are an orthonormal basis of $\mathcal{T}$ and eigenvectors of $A$, and (d) $A$ and $I-A$ are strictly positive on $\%$. Now we define $n\left(f_{k}\right)=a\left(f_{k}\right)^{*} a\left(f_{k}\right)$, $k=1, \ldots, 2 p$ and let

$$
x=n\left(f_{1}\right) \cdots \cdot n\left(f_{p}\right) \cdot\left(I-n\left(f_{p+1}\right)\right) \cdots\left(I-n\left(f_{2 p}\right)\right) .
$$

By (ii) above, we have that $x$ is in $I_{p}$ since $y$ is, i.e., the left (right) ordering procedures will result in polynomials of minimal degree $p$ in creators (destructors) respectively.

Finally, letting $\lambda_{1}, \ldots, \lambda_{2 p}$ be the eigenvalues corresponding to $f_{1}, \ldots, f_{2 p}$ we have

$$
\omega_{A}(x)=\lambda_{1} \cdot \lambda_{2} \cdots \cdot \lambda_{p} \cdot\left(1-\lambda_{p+1}\right) \cdot\left(1-\lambda_{p+2}\right) \cdots\left(1-\lambda_{2 p}\right)>0 .
$$

Thus $\Pi_{A}(x) \neq 0$ or $\Pi_{A}\left({ }_{n} I_{m}\right) \neq 0$, giving a contradiction. Done.

In this section, we have characterized factor states of $\mathscr{Q}^{\circ}(\mathscr{K})$ which were obtained from gauge invariant generalized free states of $\mathscr{Q}(\mathcal{K})$. In the following section, we turn our attention to the question of quasi-equivalence for two such states of $\mathbb{Q}^{\circ}(\mathcal{K})$.

4. Quasi-equivalence of representations. In this section, we wish to prove

THEOREM. Let $\omega_{A}^{\circ}$ and $\omega_{B}^{\circ}$ be factor states of $\mathbb{Q}^{\circ}(\mathcal{F})$, that is, $\operatorname{Tr} A(I-A)$ $=\operatorname{Tr} B(I-B)=\infty$. Then $\omega_{A}^{\circ} \sim_{q} \omega_{B}^{\circ}$ if and only if $A^{1 / 2}-B^{1 / 2}$ and $(I-A)^{1 / 2}$ $-(I-B)^{1 / 2}$ are of Hilbert-Schmidt class.

To do so, we will make use of the fact that $\left\|\omega_{A}^{\circ}-\omega_{B}^{\circ}\right\|<2$ implies $\omega_{A}^{\circ} \sim_{q} \omega_{B}^{\circ}$, by the theorem below and Proposition 3.25. (See (i) if and only if (iv).)

TheOREM 4.1 [4, THEOREM 4.5]. Let $\mathbb{Q}=\overline{U_{n} \mathbb{Q}_{n}}$ and let $\Pi_{1}$ and $\Pi_{2}$ be two factor representations of $\mathbb{Q}$ such that $\operatorname{ker} \Pi_{1}=\operatorname{ker} \Pi_{2}$. Let $\omega_{1}$ and $\omega_{2}$ be vector spaces of $\Pi_{1}$ and $\Pi_{2}$ respectively. Then the following statements are equivalent:

(i) $\Pi_{1}$ and $\Pi_{2}$ are quasi-equivalent.

(ii) For all $\varepsilon>0$ there is an integer $r$ such that

$$
\left|\omega_{1}(x)-\omega_{2}(x)\right|<\varepsilon\left\|\Pi_{1}(x)\right\| \text { for all } x \text { in } \mathbb{Q}_{r}^{c} .
$$

(iii) For all $\varepsilon>0$ there is a finite-dimensional $*$-algebra $\mathscr{B} \subset \mathbb{Q}$ containing $e$ such that 


$$
\left|\omega_{1}(x)-\omega_{2}(x)\right|<\varepsilon\left\|\Pi_{1}(x)\right\| \text { for all } x \text { in } \mathfrak{B}^{c} .
$$

(iv) There exists a finite-dimensional *-algebra $\subset \subset \mathbb{Q}$ containing $e$ such that $\operatorname{Sup}\left\{\left|\omega_{1}(x)-\omega_{2}(x)\right|: x\right.$ in $\left.\mathcal{C}^{c},\left\|\Pi_{1}(x)\right\| \leqslant 1\right\}<2$.

In previous work ([12], [14]) projection states of the CAR and GICAR algebras were successfully analysed by the device of "doubling" the Hilbert space (from $\mathcal{K}$ to $\mathscr{T} \oplus \Re$ ). Here, we find

$$
E_{A}=\left(\begin{array}{cc}
A & A^{1 / 2}(I-A)^{1 / 2} \\
A^{1 / 2}(I-A)^{1 / 2} & I-A
\end{array}\right)
$$

is a projection, i.e., $E_{A}=E_{A}^{*} E_{A}$, and the restriction of $\omega_{E_{A}}$ (a state of $\mathscr{Q}(\mathscr{K} \oplus \mathscr{K}))$ to $\mathscr{Q}(\mathcal{K} \oplus 0)$ is simply $\omega_{A}$. Our. ability to analyse projection states will suggest a method of proof.

Theorem 4.2 [12, Theorem 2.8 AND Lemma 4.5]. Let $E$ and $F$ be projections on a Hilbert space $\mathcal{K}_{0}$ Let $\omega_{E}$ and $\omega_{F}$ be the associated gauge invariant generalized free states of $\mathfrak{Q}(\mathcal{F})$. Then $\omega_{E} \sim \omega_{F}$ if and only if $E-F$ is Hilbert-Schmidt class. Further, for $E_{A}$ and $E_{B}$ as above, $E_{A}-E_{B}$ is Hilbert-Schmidt class if and only if $A^{1 / 2}-B^{1 / 2}$ and $(I-A)^{1 / 2}-(I-B)^{1 / 2}$ are Hilbert-Schmidt class.

The above theorem indicates that projections nearby in Hilbert-Schmidt norm produce equivalent states. Hence we will attempt to construct a path from $E_{A}$ to $E_{B}$ with an appropriate Hilbert-Schmidt continuity property; this will give a path from $\omega_{A}^{\circ}$ to $\omega_{B}^{\circ}$ as the following diagram indicates:

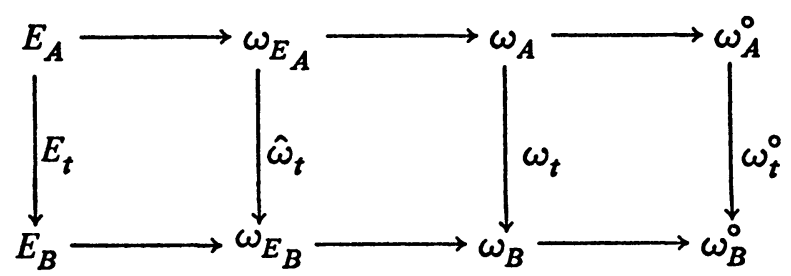

Figure 2. Deformation diagram

where $\hat{\omega}_{t}$ is defined to be $\omega_{E_{t}}$, the state obtained from $E_{t}$ in the usual way, $\omega_{t}=\hat{\omega}_{t} \mid \mathcal{Q}(\mathcal{T} \oplus 0)$, and $\omega_{t}^{\circ}=\omega_{t} \mid \mathscr{Q}^{\circ}(\mathcal{T} \oplus 0)$. Finally, the continuity properties of the original path will carry over to the restriction paths, and the quasiequivalence of $\omega_{A}^{\circ}$ and $\omega_{B}^{\circ}$ will follow from these. We begin, then, by studying pairs of projections, in order to construct the desired path $E_{t}$.

LEMMA 4.3. Let $E$ and $F$ be projections on a Hilbert space. Then

(i) $E-F$ is selfadjoint. 
(ii) If $E-F$ is Hilbert-Schmidt, then it is compact with discrete spectrum contained in $[-1,1]$.

Proof. $E-F$ is selfadjoint since $E$ and $F$ are, and $E-F$ is HilbertSchmidt if and only if $\operatorname{Tr}(E-F)^{2}<\infty$ which implies $E-F$ is compact, and hence has pure point spectrum. Further

$$
(f,(E-F) f)=(f, E f)-(f, F f)=\|E f\|^{2}-\|F f\|^{2}
$$

which implies $-1<(f,(E-F) f) \leqslant 1$. Done.

Lemma 4.4. Let $E$ and $F$ be projections on a Hilbert space $\mathcal{K}$, such that $E-F$ is Hilbert-Schmidt class. Then we can write

so that

$$
E=E^{(0)}+E^{(1)}, \quad F=F^{(0)}+F^{(1)}
$$

(i) $E^{(0)}, E^{(1)}, F^{(0)}, F^{(1)}$ are projections and $E^{(0)} E^{(1)}=E^{(1)} F^{(1)}=F^{(0)} F^{(1)}$ $=0$.

(ii) The dimensions of the ranges of $E^{(1)}$ and $F^{(1)}$ are finite.

(iii) $\left\|E^{(0)}-F^{(0)}\right\|<1$.

Proof. Since $E-F$ is selfadjoint and compact, it follows that differing nonzero eigenvalues of $E-F$ correspond to mutually orthogonal finitedimensional eigenspaces. That is, defining $\mathscr{K}_{\alpha}=\left\{f \in \Re_{i}(E-F) f=\alpha f\right\}$ and $n_{\alpha}=\operatorname{dim}\left(\Re_{\alpha}\right)$, we have $\Re_{\alpha} \perp \Re_{\alpha}, \alpha \neq \alpha^{\prime}$, and $n_{\alpha}<\infty$ for all $\alpha \neq 0$ in $[-1,1]$. Now we observe that, for all $f \in \Re_{ \pm 1}$ we have $(E-F) f= \pm f$ implies

$$
\begin{gathered}
\|E f\|=1 \text { and }\|F f\|=0, \\
\|F f\|=1 \text { and }\|E f\|=0,
\end{gathered}
$$

respectively. That is,

$$
f \in\left\{\begin{array}{l}
\operatorname{Ran} E \cap(\operatorname{Ran} F)^{\perp}=\mathscr{K}_{1}, \\
\operatorname{Ran} F \cap(\operatorname{Ran} E)^{\perp}=\mathscr{K}_{-1},
\end{array}\right.
$$

respectively. Hence we define $E^{(1)}, F^{(1)}$ as the projections on $\varkappa_{+1}, \Re_{-1}$, respectively, and $E^{(0)}, F^{(0)}$ as $E-E^{(1)}, F-F^{(1)}$, respectively. Since we have split off the spaces where $(E-F) f= \pm f$, and clearly $|(g,(E-F) g)|<1$ for all $g \in \mathscr{K}$, we observe $\left\|E^{(0)}-F^{(0)}\right\|<1$, giving (iii). Finally, by the definitions of $E^{(0)}, E^{(1)}, F^{(0)}, F^{(1)}$ and the first remark of the proof, we have (i) and (ii). Done.

LEMMA 4.5. See, e.g., $\left[14, p\right.$. 74]. Let $E^{(0)}$ and $F^{(0)}$ be projections on a Hilbert space $\Re$ such that $\left\|E^{(0)}-F^{(0)}\right\|<1$ and $E^{(0)}-F^{(0)}$ is Hilbert-Schmidt class. Then we can decompose $\mathcal{K}_{\mathrm{C}}$ into orthogonal subspaces $\mathcal{K}_{u_{i}}, i=1,2, \ldots$, such that 
(i)(a) The $\mathscr{K}_{u_{1}}$ are one-dimensional (corresponding to independent vectors of the eigenspace $\mathcal{K}_{0}$ of $\left.E^{(0)}-F^{(0)}\right)$, or

(b) the $\mathcal{K}_{ \pm u_{i}}$ are $2 n$-dimensional (corresponding to $n$ pairs of nonzero eigenvalues $\pm u_{i}$ of $E^{(0)}-F^{(0)}$ with $0<u_{i}<1$ and $n$ the finite multiplicity of $\left.u_{i}\right)$.

(ii) The projections $E^{(0)}$ and $F^{(0)}$ are reduced by the $\mathcal{K}_{\mu_{i}}$.

(iii) The projections $E_{i}=E^{(0)} \mid \mathcal{K}_{u_{i}}$ and $F_{i}=F^{(0)} \mid \mathcal{K}_{u_{i}}$ are

(a) rank one or zero for $u_{i}=0$,

(b) rank one for $u_{i} \neq 0$.

Proof. For convenience, we reproduce the argument in [14]. For (i)(b), let $0<|u|<1$ and suppose $\left(E^{(0)}-F^{(0)}\right) f=u f$, with $f \neq 0$. Let $g=-\left(E^{(0)}\right.$ $\left.-F^{(0)}-u I\right) E^{(0)} f$. This is (1) an eigenvector of $E^{(0)}-F^{(0)}$ and (2) nonzero since

$$
\begin{aligned}
\left(E^{(0)}-F^{(0)}\right) g & =-\left(E^{(0)}-F^{(0)}\right)^{2} E^{(0)} f+u\left(E^{(0)}-F^{(0)}\right) E^{(0)} f \\
& =-u\left(-E^{(0)}+F^{(0)}+u I\right) E^{(0)} f=-u g
\end{aligned}
$$

and

$$
\begin{aligned}
\left(E^{(0)}-F^{(0)}\right) f=u f & \text { implies } E^{(0)} f=F^{(0)} f+u f \\
& \text { implies } g=-u(1-u) f+2 u F^{(0)} f ;
\end{aligned}
$$

hence if $g=0$, either $u=0$ or $F^{(0)} f=(1-u) f / 2$, in which case $u=1$. Since either contradicts the assumption $0<u<1$, we have $g \neq 0$. Now note that

$$
\begin{aligned}
(f, g) & =-\left(f,\left(E^{(0)}-F^{(0)}-u I\right) E^{(0)} f\right) \\
& =-\left(\left(E^{(0)}-F^{(0)}-u I\right) f, E^{(0)} f\right)=\left(u f-u f, E^{(0)} f\right)=0 ;
\end{aligned}
$$

hence $f \perp g$. Choosing mutually orthogonal vectors in $\varkappa_{u_{i}}$ until the space is exhausted, we obtain $n$ pairs of eigenvectors, corresponding to eigenvalues $\pm u$, where $n$ is the finite multiplicity of $u_{i}$. Hence $K_{ \pm u_{l}}$ is $2 n$-dimensional, completing (i)(b). For (i)(a), we note that an orthonormal basis for the zero eigenspace of $E^{(0)}-F^{(0)}$ gives the desired one-dimensional spaces.

To show (ii) for the case $0<u_{i}<1$, we solve $\left(E^{(0)}-F^{(0)}\right) f_{i}=u_{i} f_{i}$ for $E^{(0)} f$ and substitute in $g_{i}=-\left(E^{(0)}-F^{(0)}-u_{i} I\right) E^{(0)} f_{i}$ to obtain $E^{(0)} f_{i}$ $=\frac{1}{2}\left(1+u_{i}\right) f_{i}+\left(1 / 2 u_{i}\right) g_{i}$ and $F^{(0)} f_{1}=\frac{1}{2}\left(1-u_{i}\right) f_{i}+\left(1 / 2 u_{i}\right) g_{i}$. If $u_{i}=0$, we note $E^{(0)} f=F^{(0)} f$, and thus $\left[E^{(0)}\left|\mathcal{K}_{0}, F^{(0)}\right| \mathcal{K}_{0}\right]=0$. Hence the orthonormal basis for $\mathcal{K}_{0}$ may be chosen to diagonalize simultaneously $E^{(0)}$ and $F^{(0)}$, completing (ii). 
Finally, for (iii) we argue that for $0<u_{i}<1$ the $E_{i}, F_{i}$ are rank one projections since

$$
E_{i}=\left\{\begin{array}{l}
0 \mid \mathcal{K}_{u_{i}} \\
I \mid \mathcal{K}_{u_{i}}
\end{array} \text { or } \quad F_{i}=\left\{\begin{array}{l}
0 \mid \mathcal{K}_{u_{l}} \\
I \mid \mathcal{K}_{u_{i}}
\end{array}\right.\right.
$$

are precluded by the inequality $0<u_{i}<1$, together with (1) and (2). For $u_{i}=0$, the basis chosen above consists of eigenvectors for both $E^{(0)}$ and $F^{(0)}$; since they are projections the eigenvalues are zero or one, hence the corresponding $E_{i}, F_{i}$ are rank zero or one respectively-completing (iii) and the lemma.

We are now ready to construct a path from $E^{(0)}$ to $F^{(0)}$, by rotation.

LEMMA 4.6. Let $E^{(0)}, F^{(0)}$ be as above. There is a path $E_{t}^{(0)}, t \in[-1,1]$, such that $E_{-1}^{(0)}=E^{(0)}, E_{1}^{(0)}=F^{(0)}$, and $\left\|E_{r}^{(0)}-E_{s}^{(0)}\right\|_{\text {H.S. }} \rightarrow 0$ as $r \rightarrow s$.

Proof. For $i$ such that $u_{i} \neq 0$, we have $E_{i}, F_{i}$ rank one. Hence there are vectors $h_{i}, k_{i}$ such that $\operatorname{Ran}\left(E_{i}\right)=\operatorname{span}\left\{h_{i}\right\}, \operatorname{Ran}\left(F_{i}\right)=\operatorname{span}\left\{k_{i}\right\}$. An easy computation shows that for such rank one operators, $\left\|E_{i}-F_{i}\right\|_{\text {H.S. }}^{2}=2$ $-2\left|\left(h_{i}, k_{i}\right)\right|^{2}$. Since

$$
\frac{1}{\left\|f_{i}\right\|^{2}}\left(f_{i},\left(E_{i}-F_{i}\right)^{2} f_{i}\right)=\frac{1}{\left\|g_{i}\right\|^{2}}\left(g_{i},\left(E_{i}-F_{i}\right)^{2} g_{i}\right)=u_{i}^{2}
$$

we have $\left\|E_{i}-F_{i}\right\|_{\mathrm{H} . \mathrm{S} .}^{2}=2 u_{i}^{2}=2-2\left|\left(h_{i}, k_{i}\right)\right|^{2}$, and letting $\left|\left(h_{i}, k_{i}\right)\right|^{2}=\cos ^{2} \theta_{i}$, we get $u_{i}=\sin \theta_{i}$. As shown in the figure, we rotate $k_{i}$ into $h_{i}$ by varying the angle from, e.g., $(t+1) \theta_{i} / 2 \rightarrow \theta_{i}, t \in[-1,1]$. Defining $k_{i, t}$ in the obvious way, we then define $E_{i, t}$ to be the projection onto $k_{i, t}$. Then

$$
\left\|E_{i, r}-E_{i, s}\right\|_{\mathrm{H} . \mathrm{S} .}^{2}=2-2 \cos ^{2} \frac{(r-s)}{2} \theta_{i}=\sin ^{2} \frac{(r-s)}{2} \theta_{i} ;
$$

clearly, we have $E_{i,-1}=E_{i}, E_{i, 1}=F_{i}$ and $\left\|E_{i, r}-E_{i, s}\right\|_{\text {H.S. }}^{2} \rightarrow 0$ as $r \rightarrow s$. Now define

$$
E_{r}^{(0)}=E^{(0)}\left|\mathcal{K}_{0}+\sum_{\left\{i: u_{i} \neq 0\right\}} E_{i, r}=F^{(0)}\right| \mathcal{K}_{0}+\sum_{\left\{i: u_{i} \neq 0\right\}} E_{i, r} .
$$

Then $E_{-1}^{(0)}=E^{(0)}, E_{1}^{(0)}=F^{(0)}$ and

$$
\left\|E_{r}^{(0)}-E_{s}^{(0)}\right\|_{\text {H.S. }}^{2}=\sum_{i=1}^{\infty} 2 \sin ^{2} \frac{(r-s)}{2} \theta_{i} .
$$

Since $E^{(0)}-F^{(0)}$ is Hilbert-Schmidt, $\sum_{i=1}^{\infty} u_{i}^{2}=\sum_{j=1}^{\infty} \sin ^{2} \theta_{i}<\infty$. Further, for $0<x<\pi / 2,2 x / \pi<\sin x<x$ and $4 x^{2} / \pi^{2}<\sin ^{2} x<x^{2}$, so $\sum_{i=1}^{\infty} \sin ^{2} \theta_{i}<\infty$ implies $\sum_{i=1}^{\infty} \theta_{i}^{2}=M<\infty$. 
Finally,

$$
\left\|E_{r}^{(0)}-E_{s}^{(0)}\right\|_{\text {H.S. }}^{2}=\sum_{i=1}^{\infty} 2 \sin ^{2} \frac{(r-s)}{2} \theta_{i}<\frac{(r-s)^{2}}{2} M \rightarrow 0
$$

as $r \rightarrow s$. Done.

For convenience we state

LEMMA 4.7 [12, LEMMA 4.1]. Let $A$ and $B$ be positive operators on $a$ Hilbert space \%. Then

$$
\left\|A^{1 / 2}-B^{1 / 2}\right\|_{\mathrm{H} . \mathrm{S} .}^{2} \leqslant\|A-B\|_{\mathrm{TR} .}
$$

LEMMA 4.8. Let $E$ and $F$ be projections on a Hilbert space \%, with $E-F$ Hilbert-Schmidt class. Define $E=E^{(0)}+E^{(1)}, F=F^{(0)}+F^{(1)}$ as in Lemma 4.4.Then there are operators $E_{t}, t \in[-2,2]$, such that

(i) $E_{-2}=E, E_{2}=F$.

(ii) $0<E_{t}<I$.

(iii) $\left\|E_{r}-E_{s}\right\|_{H . S} \rightarrow 0$ as $r \rightarrow s$, for all $r, s$ in $[-1,1]$.

(iv) $\left\|E_{r}^{1 / 2}-E_{s}^{1 / 2}\right\|_{\text {H.S. }} \rightarrow 0,\left\|\left(I-E_{r}\right)^{1 / 2}-\left(I-E_{s}\right)^{1 / 2}\right\|_{\text {H.S. }} \rightarrow 0$ as $r \rightarrow s$, for all $r, s$ in $[-2,1]$ or $[1,2]$.

Proof. We construct paths, with the above continuity properties as follows:

$$
E_{t}= \begin{cases}E^{(0)}-(t+1) E^{(1)}, & t \in[-2,-1], \\ E_{t}^{(0)}, & t \in[-1,1], \\ F^{(0)}+(t-1) F^{(1)}, & t \in[1,2],\end{cases}
$$

with $E_{t}^{(0)}$ as in the previous lemma, whose conclusion gives (ii). First, we note the $E_{t}$ are clearly positive by definition giving (i). Now, applying Lemma 4.7 we obtain, for $r, s \in[-2,-1]$,

$$
\begin{aligned}
\left\|E_{r}^{1 / 2}-E_{s}^{1 / 2}\right\|_{\mathrm{H} . S .}^{2} & \leqslant\left\|E_{r}-E_{s}\right\|_{\mathrm{TR} .} \\
& =\left\|(s-r) E^{(1)}\right\|_{\mathrm{TR} .}=|(s-r)| n_{1} \rightarrow 0 \text { as } r \rightarrow s,
\end{aligned}
$$

recalling $n_{1}$ to be the (finite) dimension of the range of $E^{(1)}$. Likewise, we have

$$
\begin{aligned}
\left\|\left(I-E_{r}\right)^{1 / 2}-\left(I-E_{s}\right)^{1 / 2}\right\|_{\mathrm{H} . S .}^{2} & \leqslant\left\|\left(I-E_{r}\right)-\left(I-E_{s}\right)\right\|_{\mathrm{TR} .}=\left\|E_{r}-E_{s}\right\|_{\mathrm{TR} .} . \\
& =\|(s-r) \mid n_{1} \rightarrow 0 \text { as } r \rightarrow s .
\end{aligned}
$$

The identical argument for the $F_{r}$ yields, for $r, s \in[1,2]$, 


$$
\begin{array}{r}
\left\|F_{r}^{1 / 2}-F_{s}^{1 / 2}\right\|_{H . S .} \rightarrow 0 \text { as } r \rightarrow s, \\
\left\|\left(I-F_{r}\right)^{1 / 2}-\left(I-F_{s}\right)^{1 / 2}\right\|_{H . S .} \rightarrow 0 \text { as } r \rightarrow s .
\end{array}
$$

Hence, we have (iii). Done.

SUMmary. We have a path from $E$ to $F$ as shown below.

$$
\begin{aligned}
& E=E^{(0)}+E^{(1)} \longrightarrow E^{(0)} \\
& F=F^{(0)}+F^{(1)} \longleftarrow F^{(0)}
\end{aligned}
$$

FIGURE 3. Projection deformation

We are now ready to show the inherited state norm continuity. We will need, however, the following results of [12].

TheOREM 4.9 [12, THEOREM 2.6]. Suppose $\omega_{E}$ and $\omega_{F}$ are pure generalized free states of $\mathbb{Q}(\mathcal{K})$. Let $\alpha_{1}=\operatorname{det}(I-E(I-F) E)$, ard $\alpha_{2}=\operatorname{det}(I-(I-E) F(I$ $-E))$, and $\alpha=\min \left(\alpha_{1}, \alpha_{2}\right)$. Then $\left\|\omega_{E}-\omega_{F}\right\|=2(1-\alpha)^{1 / 2}$.

LEMMA 4.10 [12, LEMMA 4.7]. Let $\omega_{A}$ and $\omega_{B}$ be gauge invariant generalized free states of $\mathcal{Q}(\mathcal{H})$. Suppose $0<\varepsilon<2$. Then if $\left\|A^{1 / 2}-B^{1 / 2}\right\|_{\text {H.S. }}<\varepsilon / 12$ and $\left\|(I-A)^{1 / 2}-(I-B)^{1 / 2}\right\|_{H . S .}<\varepsilon / 12$, then $\left\|\omega_{A}-\omega_{B}\right\|<\varepsilon$.

Now we show

LEMMA 4.11. Let $E_{A}, E_{B}$ be projections on $\nVdash \oplus \Re$, defined as above. Suppose $E_{A}-E_{B}$ is Hilbert-Schmidt class. We define $E_{t}$ as in Lemma 4.8, setting $E_{A}=E$ and $E_{B}=F$, and a gauge invariant generalized free state of $\mathbb{Q}(\mathcal{T} \oplus \mathcal{K})$, $\hat{\omega}_{t}$ by the formula $\hat{\omega}_{t}=\omega_{E_{t}}$. Also, we define

$$
\omega_{t}=\omega_{t}\left|\mathbb{Q}(\mathcal{K} \oplus 0), \quad \omega_{t}^{\circ}=\omega_{t}\right| \mathscr{Q}^{\circ}(\mathcal{K} \oplus 0) .
$$

Then

(i) $\omega_{-2}^{\circ}=\omega_{A}^{\circ}$ and $\omega_{2}^{\circ}=\omega_{B}^{\circ}$.

(ii) $\left\|\omega_{r}^{\circ}-\omega_{s}^{\circ}\right\| \rightarrow 0$ as $r \rightarrow s$, for all $r, s \in[-2,2]$.

Proof. For (i), we simply observe that $\hat{\omega}_{-2}=\omega_{E_{-2}}=\omega_{E_{A}}$ and

$$
\omega_{-2}=\hat{\omega}_{-2}\left|\mathscr{Q}(\mathcal{K} \oplus 0)=\omega_{A}, \quad \omega_{-2}^{\circ}=\hat{\omega}_{-2}\right| Q^{\circ}(\Re \oplus 0)=\omega_{A}^{\circ} .
$$

Likewise, $\omega_{2}^{\circ}=\omega_{B}^{\circ}$. For (ii) we first consider $r, s \in[-1,1]$. Then by Theorem 4.9 above, we have

$$
\left\|\hat{\omega}_{r}-\hat{\omega}_{s}\right\|=\left\|\omega_{E_{r}}-\omega_{E_{s}}\right\|=2(1-\alpha)^{1 / 2}
$$


with

$$
\alpha=\min \left(\operatorname{det}\left(I-\left(I-E_{r}\right) E_{s}\left(I-E_{r}\right)\right), \operatorname{det}\left(I-E_{r}\left(I-E_{s}\right) E_{r}\right)\right) .
$$

But since $\operatorname{det}(I-A) \geqslant 1-\operatorname{Tr}(A)$ for $0 \leqslant A \leqslant I$ we obtain

$$
\alpha \geqslant \operatorname{det}\left(I-\left(I-E_{r}\right) E_{s}\left(I-E_{r}\right)\right) \geqslant 1-\operatorname{Tr}\left(\left(I-E_{r}\right) E_{s}\left(I-E_{r}\right)\right) .
$$

By positivity of $E_{r}\left(I-E_{s}\right) E_{r}$ we have

$$
\begin{aligned}
\alpha & \geqslant 1-\operatorname{Tr}\left(\left(I-E_{r}\right) E_{s}\left(I-E_{r}\right)+E_{r}\left(I-E_{s}\right) E_{r}\right) \\
& =1-\operatorname{Tr}\left(\left(E_{r}-E_{s}\right)^{2}\right)=1-\left\|E_{r}-E_{s}\right\|_{\text {H.S. }}^{2}
\end{aligned}
$$

Hence, for all $r, s \in[-1,1],\left\|\hat{\omega}_{r}-\hat{\omega}_{s}\right\|=2(1-\alpha)^{1 / 2} \leqslant 2\left\|E_{r}-E_{s}\right\|_{\text {H.S. }} \rightarrow 0$ as $r \rightarrow s$, by Lemma 4.8. Now consider $r, s \in[-2,-1]$ or $r, s \in[1,2]$. Again $\left\|\hat{\omega}_{r}-\hat{\omega}_{s}\right\|=\left\|\omega_{E_{r}}-\omega_{E_{s}}\right\|$ and from Lemma 4.8(iii), we have $\left\|E_{r}^{1 / 2}-E_{s}^{1 / 2}\right\|_{H . S}$. $\rightarrow 0$ and $\left\|\left(I-E_{r}\right)^{1 / 2}-\left(I-E_{s}\right)^{1 / 2}\right\|_{\text {H.S. }} \rightarrow 0$. But by direct application of Lemma 4.10, these imply $\left\|\omega_{E_{r}}-\omega_{E_{s}}\right\| \rightarrow 0$ as $r \rightarrow s$. Finally, $\left\|\hat{\omega}_{r}-\hat{\omega}_{s}\right\|$ $\geqslant\left\|\omega_{r}-\omega_{s}\right\| \geqslant\left\|\omega_{r}^{\circ}-\omega_{s}^{\circ}\right\|$ by the properties of restriction. Thus $\left\|\omega_{r}^{\circ}-\omega_{s}^{\circ}\right\|$ $\rightarrow 0$ as $r \rightarrow s$, for all $r, s \in[-2,2]$, completing (ii). Done.

Of use in proving our final results in this section will be

THEOREM 4.12 [12, THEOREM 5.1]. Every gauge invariant generalized free state of $\mathbb{Q}(\Re)$ is a factor state. Two gauge invariant generalized free states $\omega_{A}$ and $\omega_{B}$ are quasi-equivalent if and only if the operators $A^{1 / 2}-B^{1 / 2}$ and $(I-A)^{1 / 2}$ - $(I-B)^{1 / 2}$ are of Hilbert-Schmidt class.

We will also use the following two lemmas.

LEMMA 4.13 [12, LEMMA 2.3]. Suppose $\omega_{1}$ and $\omega_{2}$ are even states of $\mathfrak{Q}(\mathcal{K})$ and Th is a finite-dimensional subspace of $\nVdash_{0}$ Then

$$
\left\|\omega_{1}\left|\mathbb{Q}(\Re)^{c}-\omega_{2}\right| \mathbb{Q}(\Re)^{c}\right\|=\left\|\omega_{1}\left|\mathbb{Q}\left(\Re^{\perp}\right)-\omega_{2}\right| \mathbb{Q}\left(\Re^{-\perp}\right)\right\| .
$$

REMARK. We note that generalized free states are defined to be even.

LEMMA 4.14 [12, LEMMA 4.5]. Let $A$ and $B$ be operators on $a$ Hilbert space $\mathcal{K}$ such that $0 \leqslant A \leqslant I$ and $0 \leqslant B \leqslant I$. Then $E_{A}-E_{B}$ is a Hilbert-Schmidt class operator if and only if the operators $A^{1 / 2}-B^{1 / 2}$ and $(I-A)^{1 / 2}-(I-B)^{1 / 2}$ are of Hilbert-Schmidt class.

We are now ready to prove

THEOREM 4.15. Let $A$ and $B$ be operators with point spectrum such that $0<A<I, 0<B<I$ and $\operatorname{Tr} A(I-A)=\operatorname{Tr} B(I-B)=\infty$. Let $\omega_{A}^{\circ}$ and $\omega_{B}^{\circ}$ be the corresponding factor series of $\mathbb{Q}^{\circ}(\mathcal{K})$. Then $\omega^{\circ} \tau_{q} \omega_{B}^{\circ}$ if and only if $A^{1 / 2}-B^{1 / 2}$ and $(I-A)^{1 / 2}-(I-B)^{1 / 2}$ are of Hilbert-Schmidt class. 
Proof. Sufficiency. Suppose $A^{1 / 2}-B^{1 / 2}$ and $/$ or $(I-A)^{1 / 2}-(I-B)^{1 / 2}$ are not Hilbert-Schmidt. Then by Theorem 4.12 above, we have $\omega_{A} \uparrow_{q} \omega_{B}$, where $\omega_{A}$ and $\omega_{B}$ are the gauge invariant generalized free states of the whole CAR, $\mathscr{Q}(\mathcal{K})$. Hence, for all finite-dimensional $\Re \subset \mathcal{K}$, we have

$$
\left\|\left(\omega_{A}-\omega_{B}\right) \mid \mathscr{Q}(\Re)^{c}\right\|=2
$$

by, e.g., Lemma 3.23 above. Now, by Lemma 3.9, $\mathbb{Q}^{\circ}(\pi)^{c_{0}} \subset \mathbb{Q}^{\circ}\left(\pi^{\perp}\right)$, so we have

$$
\left\|\left(\omega_{A}^{\circ}-\omega_{B}^{\circ}\right)\left|\mathscr{Q}^{\circ}(\Re)^{c_{0}}\|\geqslant\|\left(\omega_{A}^{\circ}-\omega_{B}^{\circ}\right)\right| \mathscr{Q}^{\circ}\left(\Re^{\perp}\right)\right\|
$$

and from the definitions, we conclude

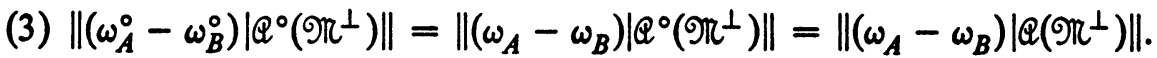

Finally from Lemma 4.13 we have

$$
\left\|\left(\omega_{A}-\omega_{B}\right)\left|\mathbb{Q}\left(\Re^{\perp}\right)\|=\|\left(\omega_{A}-\omega_{B}\right)\right| \mathbb{Q}(\mathscr{T})^{c}\right\|,
$$

since generalized free states are necessarily even. Combining (1) through (4) gives

$$
\left\|\left(\omega_{A}^{\circ}-\omega_{B}^{\circ}\right) \mid \mathscr{Q}^{\circ}(\Re)^{c_{0}}\right\| \geqslant 2 ;
$$

hence by Theorem 4.1 and Proposition 3.24 we conclude $\omega_{A}^{\circ} \uparrow_{q} \omega_{B}^{\circ}$. Done with sufficiency.

Necessity. Suppose $A^{1 / 2}-B^{1 / 2}$ and $(I-A)^{1 / 2}-(I-B)^{1 / 2}$ are HilbertSchmidt. It follows from above that $E_{A}-E_{B}$ is Hilbert-Schmidt. Now we invoke the path $\omega_{t}$ of Lemma 4.11. Recall $\left\|\omega_{r}-\omega_{s}\right\| \rightarrow 0$ as $r \rightarrow s$, for all $r, s \in[-2,2]$, and $\omega_{-2}=\omega_{A}, \omega_{2}=\omega_{B}$. It follows that we can choose a partition of $[-2,2]$, say $\left\{t_{k}\right\}, 1 \leqslant k \leqslant N$, such that $t_{1}=-2$ and $t_{N}=2$ and $\left\|\omega_{t_{k+1}}-\omega_{t_{k}}\right\|<2,1<k<N-1$. Since the $\omega_{t}$ are restrictions of the gauge invariant generalized free states $\omega_{E_{t}}$, they are likewise generalized free states, and hence factors, by Theorem 4.12 above. Now observe that since $\omega_{t_{1}}=\omega_{A}$ and $\operatorname{Tr} A(I-A)=\infty, \omega_{t_{1}}$ is not type I by Proposition 3.22. But by Lemma 3.23, since $\left\|\omega_{t_{k+1}}-\omega_{t_{k}}\right\|<2$, we have $\omega_{t_{k+1}} \tau_{q} \omega_{t_{k}}, 1 \leqslant k \leqslant N-1$. Thus the $\omega_{t_{k}}$ are not type I, and the positive operators $A_{t_{k}}$ defined by the two-point function of $\omega_{t_{k}}$ have the property that $\operatorname{Tr} A_{t_{k}}\left(I-A_{t_{k}}\right)=\infty$, again by Proposition 3.22. We can then conclude, by Theorem 3.24, that $\omega_{i_{k}}^{\circ}$ is a factor state of $\mathbb{Q}^{\circ}(\mathcal{K}), 1 \leqslant k \leqslant N$. Finally, we have $\omega_{-2}^{\circ}=\omega_{A}^{\circ}, \omega_{2}^{\circ}=\omega_{B}^{\circ}$ and $\| \omega_{t_{k+1}}^{\circ}$ $-\omega_{t_{k}}^{\circ} \|<2$, the latter by restriction, $1 \leqslant k \leqslant N-1$. Thus $\omega_{t_{k}}^{0} \tau_{q} \omega_{t_{k+1}}^{0}, 1$ $\leqslant k \leqslant N-1$ and since quasi-equivalence is an equivalence relation, $\omega_{i_{1}}^{\circ}$ $\tau_{q} \omega_{t_{N}}^{\circ}$ or $\omega_{A}^{\circ} \tau_{q} \omega_{B}^{\circ}$. Done. 
To remove the point spectrum condition, we will make use of Lemma 4.10 and the following lemma.

LEMMA 4.16 [12, LeMMA 4.4]. Let $A$ be an operator such that $0 \leqslant A \leqslant I$, and let $\varepsilon>0$. Then there exists an operator $B, 0 \leqslant B \leqslant I$, with pure point spectrum such that $A^{1 / 2}-B^{1 / 2}$ and $(I-A)^{1 / 2}-(I-B)^{1 / 2}$ are of Hilbert-Schmidt class with Hilbert-Schmidt norm less than $\varepsilon$. Furthermore, the eigenvalues of $B$ are dense in the spectrum of $A$.

TheOREM 4.17. Let $A$ and $B$ be operators such that $0<A<I, 0<B<I$ and $\operatorname{Tr} A(I-A)=\operatorname{Tr} B(I-B)=\infty$. Let $\omega_{A}^{\circ}$ and $\omega_{B}^{\circ}$ be the corresponding factor states of $\mathcal{Q}^{\circ}(\mathcal{K})$. Then $\omega_{A}^{\circ} \sim_{q} \omega_{B}^{\circ}$ if and only if $A^{1 / 2}-B^{1 / 2}$ and $(I-A)^{1 / 2}$ $-(I-B)^{1 / 2}$ are of Hilbert-Schmidt class.

Proof. Sufficiency. Same proof as Theorem 4.15, sufficiency.

Necessity. By Lemma 4.16 there exist operators $\hat{A}$ and $\hat{B}$, each with pure point spectrum, such that

$$
\begin{array}{ll}
\left\|A^{1 / 2}-\hat{A}^{1 / 2}\right\|_{\text {H.S. }}<1 / 6 & \left\|B^{1 / 2}-B^{1 / 2}\right\|_{\text {H.S. }}<1 / 6, \\
\left\|(I-A)^{1 / 2}-(I-\hat{A})^{1 / 2}\right\|_{\text {H.S. }}<1 / 6, & \left\|(I-B)^{1 / 2}-(I-B)^{1 / 2}\right\|_{\text {H.S. }}<1 / 6 .
\end{array}
$$

Further, by Lemma 4.10 the associated states of $\mathcal{Q}(\mathscr{K}), \omega_{\mathcal{A}}$ and $\omega_{B}$, have the properties $\left\|\omega_{A}-\omega_{A}\right\|<2,\left\|\omega_{B}-\omega_{B}\right\|<2$; hence, by Lemma 3.23, $\omega_{A}$ $\widetilde{\tau}_{q} \omega_{A}$ and $\omega_{B} \tau_{q} \omega_{B}$. By hypothesis and the above Proposition 3.22, $\omega_{A}$ and $\omega_{B}$ are not type $I$, and therefore $\omega_{A}$ and $\omega_{B}$ are not type I by quasi-equivalence. Thus $\operatorname{Tr} \hat{A}(I-\hat{A})=\operatorname{Tr} \hat{B}(I-\hat{B})=\infty$, which implies $\omega_{\hat{A}}^{\circ}$ and $\omega_{\hat{B}}^{\circ}$ are factor states of $Q^{\circ}(\mathcal{F})$ by Theorem 3.24. Further

$$
\begin{aligned}
\left\|A^{1 / 2}-B^{1 / 2}\right\|_{H . S .} & =\left\|A^{1 / 2}-A^{1 / 2}+A^{1 / 2}-B^{1 / 2}+B^{1 / 2}-B^{1 / 2}\right\|_{H . S .} \\
& \leqslant 1 / 6+\left\|A^{1 / 2}-B^{1 / 2}\right\|_{H . S .}+1 / 6<\infty
\end{aligned}
$$

by hypothesis. Likewise $\left\|(I-\hat{A})^{1 / 2}-(I-\hat{B})^{1 / 2}\right\|_{\text {H.S. }}<\infty$, and by the previous theorem, $\omega_{A}^{\circ} \sim_{q} \omega_{B}^{\circ}$. Hence we have $\omega_{A}^{\circ} \sim_{q} \omega_{A}^{\circ} \sim_{q} \omega_{B}^{\circ} \sim_{q} \omega_{B}^{\circ}$ or $\omega_{A}^{\circ}$ $\sim_{q} \omega_{B}^{\circ}$. Done necessity, and theorem.

\section{BIBLIOGRAPHY}

1. H. Araki, On quasifree states of CAR and Bogoliubov automorphisms, Publ. Res. Inst. Math. Sci. 6 (1970/71), 385-442. MR 45 \#4768.

2. H. Araki, and W. Wyss, Representations of the canonical anticommutation relations, Helv. Phys. Acta. 37 (1964), 136-159. MR 30 \# 1752.

3. B. M. Baker, Free states of the gauge invariant canonical anticommutation relations. II, preprint, Dalhousie Univ., Halifax, N. S., Canada.

4. O. Bratteli, Inductive limits of finite dimensional $C^{*}$-algebras, Trans. Amer. Math. Soc. 171 (1972), 195-234. MR 47 \#844. 
5. E. Balslev, J. Manuceau and A. Verbeure, Representations of anticommutation relations and Bogoliubov transformations, Comm. Math. Phys. 8 (1968), 315-326. MR 40 \#6860.

6. J. G. Glimm, On a certain class of operator algebras, Trans. Amer. Math. Soc. 95 (1960), 318-340. MR 22 \#2915.

7. S. Doplicher, R. Haag and J. E. Roberts, Fields, observables and gauge transformations. I, Comm. Math. Phys. 13 (1969), 1-23. MR 41 \# 3041.

8. - Fields, observables and gauge transformations. II, Comm. Math. Phys. 15 (1969), 173-200. MR 41 \#4922.

9. R. Haag and D. Kastler, An algebraic approach to quantum field theory, J. Mathematical Phys. 5 (1964), 848-861. MR 29 \#3144.

10. R. T. Powers, Thesis, Princeton Univ., 1967.

11. - Representations of uniformly hyperfinite algebras and their associated von Neumann rings, Ann. of Math. (2) 86 (1967), 138-171. MR 36 \# 1989.

12. R. T. Powers and E. Størmer, Free states of the canonical anticommutation relations, Comm. Math. Phys. 16 (1970), 1-33. MR 42 \# 4126.

13. D. Shale and W. F. Stinespring, States of the Clifford algebra, Ann. of Math. (2) 80 (1964), 365-381. MR 29 \#3160.

14. G. Stamatopoulos, Thesis, Univ. of Pennsylvania, 1974.

Department of Physics, University of Pennsyzvania, Philadelphia, Pennsylvania 19174

Current address: Department of Mathematics, Dalhousie University, Halifax, Nova Scotia, Canada 